\title{
Investment casting and experimental testing of heat sinks designed by topology optimization
}

Lei, Tian; Alexandersen, Joe; Lazarov, Boyan Stefanov; Wang, Fengwen; Haertel, Jan Hendrik Klaas; Sanna, Simone; Sigmund, Ole; Engelbrecht, Kurt

Published in:

International Journal of Heat and Mass Transfer

Link to article, DOI:

10.1016/j.jheatmasstransfer.2018.07.060

Publication date:

2018

Document Version

Peer reviewed version

Link back to DTU Orbit

Citation $(A P A)$ :

Lei, T., Alexandersen, J., Lazarov, B. S., Wang, F., Haertel, J. H. K., Sanna, S., Sigmund, O., \& Engelbrecht, K. (2018). Investment casting and experimental testing of heat sinks designed by topology optimization. International Journal of Heat and Mass Transfer, 127, 396-412.

https://doi.org/10.1016/j.ijheatmasstransfer.2018.07.060

\section{General rights}

Copyright and moral rights for the publications made accessible in the public portal are retained by the authors and/or other copyright owners and it is a condition of accessing publications that users recognise and abide by the legal requirements associated with these rights.

- Users may download and print one copy of any publication from the public portal for the purpose of private study or research.

- You may not further distribute the material or use it for any profit-making activity or commercial gain

- You may freely distribute the URL identifying the publication in the public portal 


\section{Investment casting and experimental testing of heat sinks designed 2 by topology optimization}

\begin{tabular}{|c|c|}
\hline 3 & $\begin{array}{l}\text { Tian Lei }^{1,3} \text {, Joe Alexandersen } \\
\text {, } \\
\text { Salvatore Doyan S. Lazarov }^{2} \text {, Fengwen Wang } \\
\end{array}$ \\
\hline 4 & \\
\hline 5 & ${ }^{1}$ Department of Energy Conversion and Storage, Technical University of Denmark, 4000 \\
\hline 6 & Roskilde, Denmark \\
\hline 7 & ${ }^{2}$ Department of Mechanical Engineering, Technical University of Denmark, 2800 Lyngby, \\
\hline 8 & Denmark \\
\hline 9 & ${ }^{3}$ Sumitomo (SHI) Cryogenics of America, Inc., 1833 Vultee St, Allentown, PA 18103, \\
\hline 10 & U.S.A. \\
\hline 11 & \\
\hline 12 & *corresponding author: kuen@dtu.dk \\
\hline 13 & \\
\hline 14 & Abstract. Topology optimization (TO) is an attractive numerical tool to obtain optimized \\
\hline 15 & engineering designs, which has been originally developed for mechanical optimization and \\
\hline 16 & extended to the area of conjugate heat transfer. With rapid developments in topology \\
\hline 17 & optimization models, promising designs have been proposed and presented recently for \\
\hline 18 & conjugate heat transfer problems. However, only a very small number of experimental \\
\hline 19 & validations of TO heat transfer devices have been reported. In this paper, investment casting \\
\hline 20 & (IC) using 3D stereolithography (SLA) printed patterns is proposed to fabricate 3D metal \\
\hline 21 & heat transfer devices designed by TO. Three heat sinks for natural convection are designed \\
\hline 22 & by a previously reported topology optimization model and five reference pin-fin heat sinks \\
\hline 23 & are devised for comparison. From those designs six heat sinks are cast in Britannia metal, \\
\hline 24 & fully reproducing the complex 3D optimized designs. It shows that SLA-assisted IC is a very \\
\hline 25 & promising technology with low cost and high accuracy for fabricating TO metal parts, which \\
\hline 26 & is not limited to heat transfer devices and can be extended to other areas such as structural \\
\hline 27 & optimization. A natural convection experimental setup is used to experimentally study the \\
\hline 28 & performance of the fabricated heat sinks. The results show that the tested TO heat sinks can \\
\hline 29 & always realize the best heat dissipation performance compared to pin-fin heat sinks, when \\
\hline 30 & operating under the conditions used for the optimization. Moreover, validation simulations \\
\hline 31 & have been conducted to investigate the temperature distribution, fluid flow pattern and local \\
\hline 32 & heat transfer coefficient for the TO and pin-fin designs, further evidencing that TO designs \\
\hline 33 & always perform better under the design conditions. In addition, the impact of heat sink \\
\hline & ited. \\
\hline
\end{tabular}

Keywords. topology optimization, heat sink, natural convection, investment casting, stereolithography printing, experimental test 
Nomenclature

\begin{tabular}{|c|c|c|c|}
\hline \multicolumn{3}{|c|}{ Variables } & 41 \\
\hline \multirow[t]{2}{*}{$A$} & \multirow[t]{2}{*}{ area } & \multirow[t]{2}{*}{$\alpha$} & Orientation angle of the heat sink with respe 42 \\
\hline & & & to gravity (polar angle) \\
\hline $\mathbf{A}_{\mathrm{m}}$ & Convective heat transfer area & $\alpha_{B}$ & Brinkman penalization coefficient 44 \\
\hline$c_{p}$ & Specific heat & $\beta$ & $\begin{array}{l}\text { Coefficient of thermal expansion, rotatikn } \\
\text { angle of the heat sink (azimuthal angle) }\end{array}$ \\
\hline $\boldsymbol{g}$ & Gravity & $\gamma$ & $\begin{array}{l}\text { Density field for topology optimization } 4(0) \\
\text { represents solid and } 1 \text { represents fluid) }\end{array}$ \\
\hline $\boldsymbol{h}$ & $\begin{array}{l}\text { Convective heat transfer } \\
\text { coefficient }\end{array}$ & $\epsilon$ & $\begin{array}{ll}\text { Emissivity } & 49 \\
& 50 \\
\end{array}$ \\
\hline $\boldsymbol{I}$ & Current & $\mu$ & Kinematic viscosity \\
\hline $\boldsymbol{k}$ & Thermal conductivity & $\rho_{0}$ & Density of the fluid \\
\hline$p$ & Pressure & $\sigma$ & Stefan-Boltzmann constant \\
\hline$Q$ & Heat transfer power & & 54 \\
\hline \multirow[t]{2}{*}{$q^{\prime \prime \prime}(x)$} & Volumetric heat generation & Subscripts & 55 \\
\hline & & $\mathrm{a}$ & Air \\
\hline \multirow[t]{2}{*}{$T$} & Temperature & Brit & Britannia metal \\
\hline & & conv & Convection \\
\hline$T_{m}$ & Metal surface temperature & eff & Effective \\
\hline$T_{0}$ & Ambient temperature & $\mathrm{f}$ & Fluid \\
\hline $\mathbf{u}$ & Fluid velocity vector & $\mathrm{m}$ & Metal \\
\hline $\mathbf{V}$ & Voltage & $\mathrm{rad}$ & Radiation \\
\hline$x_{\text {cond }}$ & Thermal conduction distance & $\mathrm{s}$ & Solid \\
\hline
\end{tabular}

\section{Introduction}

Topology optimization is a method that optimizes material distribution within a design domain for maximizing a desired objective under given constraints. TO was originally developed for structural mechanics but has since been applied to different areas such as acoustics, fluid mechanics, conjugate heat transfer, and several others [1,2]. To set up the optimization, boundary conditions, constraints and an objective function are defined first and the finite element method is usually used to model the physical problem. The design is usually improved iteratively using a variety of algorithms like density based topology optimization, level set, topological derivative, phase field and evolutionary approaches [3].

During the last decade, several studies have been presented to optimize flow, conduction, convection and conjugate heat transfer problems, which were reviewed in Refs. [4]. Bruns [5] used topology optimization to study steady-state convection-dominated heat transfer problems. Yoon [6] presented a study of 2D thermofluid topology optimization of heat dissipating structures with forced convective heat transfer. Topology optimization of heat and mass transfer problems was investigated by Marck et al. [7] and the authors presented three examples of conjugate heat transfer, including a full bi-objective optimization. Coffin and Maute [8] showed 2D and 3D topology optimization of convective heat transfer problems, using the level set method. In their study, the convection coefficient was assumed constant, but an artificial diffusive model was introduced for the fluid temperature to avoid internal cavities. Topology optimization was applied to liquid jet impingement on a cold plate in order to 
maximize heat transfer over a plate while minimizing pump work [9] and a detailed experimental evaluation of the heat sink design was presented by Dede and Liu [10]. The jet impingement design was then expanded to an array of jets fed by a topology optimized manifold that distributed equal fluid flow to each jet [11]. Koga et al. [12] used topology optimization to design a heat sink system for minimizing the pressure drop in the fluid flow and maximizing the heat dissipation effect. Dede [13] presented topology optimization of steady state convection-diffusion heat transfer problems using the commercial simulation software COMSOL Multiphysics [14]. An example of the channel topology was given for minimizing the mean temperature and total dissipated fluid power. Dede et al. [15] further fabricated a TO heat sink using metal additive manufacturing and experimentally compared its performance to convectional fin / pin-fin heat sinks. Haertel and Nellis [16] applied density-based topology optimization to heat exchanger design assuming a fully developed internal flow. The pressure drop and air-side temperature change were prescribed and the conductance of the heat exchanger was maximized. Alexandersen et al. [17] developed 2D topology optimization of heat sinks for natural convection using the steady-state incompressible Navier-Stokes equations coupled to the thermal convection-diffusion equation through the Boussinesq approximation. This work was further extended to 3D by using a large scale TO framework to design heat sinks with an order of 20-330 million state degrees of freedom in Ref. [18]. In addition, this TO tool was applied to design heat sinks for light-emitting diode lamps [19,38]. The optimized designs from Refs. [19, 38] were produced using selective laser melting of an aluminum alloy and tested compared to a comparable pin fin heat exchanger [20]. Dilgen et al. [21] applied topology optimization to a forced convection heat sink for turbulent flow in 3D and showed that complex 3D designs can be obtained. This paper presents a continuing study based on Refs. [19] and [38] and using a similar approach as Ref. [20], in which we design passive heat sinks for natural convection. However, here we cast them using the lower cost lost wax method, and implement related experimental tests, including an additional comparison with simple pin fin designs.

As mentioned above, the experimental validation of TO conjugate heat transfer devices has rarely been carried out and only six related studies, Refs. [9-12], [15] and [20], can to our knowledge be referenced, although a large number of topological designs of conjugate heat transfer problems have been presented. This is partly due to the fact that most presented topology optimization works treat rather academic problems with e.g. artificial material properties or unrealistic operating conditions, which cannot be easily converted to prototypes suitable for experiments. Another issue is that topology optimized heat transfer devices often exhibit complex shapes with fine features or complex geometries, which may be challenging or time consuming to manufacture using traditional machining methods. However, alternative manufacturing techniques such as stamping or casting could be promising for mass production of TO designs. New developing technologies such as metal additive manufacturing (AM), typically selective laser melting, thus become very attractive to fabricate 3D topology-optimized designs. A good example is the work presented by Dede et al. [15], showing that combining metal AM and TO designs is a promising path to develop new heat transfer devices. Lazarov et al. [20] also demonstrate that TO designs can outperform conventional designs for natural convection applications using AM metal heat exchangers. Although metal AM is becoming a mature technology and has been applied in specific areas including aeronautical and medical industries, there are still some limits to fabricate TO thermal devices using metal AM, such as high cost, limited material selection, potential reduction in thermal properties due to porosity in the structure and difficult post-processing. Therefore, this paper proposes investment casting assisted with stereolithography 3D printing, "SLA-assisted IC for short," to fabricate metal devices designed by topology optimization as an alternative.

Investment casting is one of the oldest manufacturing processes, which is still widely used for fabricating jewelry and industrial metal products [22]. In the traditional IC process, the wax pattern is 
shaped into the desired pattern by hand or injection molding and is then surrounded with a refractory (the investment) that sets in the mold. By heating the mold, the wax pattern melts and flows out, forming a continuous cavity. Then molten metal at the proper casting temperature is poured into the mold. After cooling, the mold is broken and the solidified metal part is removed and post-processed. Due to the usage of wax, this process is often referred to as lost wax casting, especially for jewelry production. However, shaping wax directly into a complicated TO geometry is very difficult with conventional methods. Modern 3D printing technology provides a new approach to fabricate complicated patterns precisely based on wax or castable resin [23]. The latter is a photochemically solidified material and melts out like wax during heating. As both SLA printing and investment casting exhibit high accuracy $[22,23]$, this process can precisely produce the desired topology optimized metal parts.

Compared to 3D metal AM, SLA-assisted IC has lower cost based on the authors' prototyping experience. The initial cost of the SLA printer and the casting equipment is about 5,000 USD and the casting cost of a $50 \mathrm{~cm}^{3}$ part is about 30 USD including printing and casting. As a lax comparison, a direct metal laser sintering (DMLS) device costs at least 100,000 USD and the printing cost is beyond 500 USD to prototype the same part [24]. It is anticipated that the cost of SLA assisted IC is still competitive in the mass production level. In addition, IC is a sophisticated technology and offers a wide choice of commonly used high conductive metals and alloys for heat transfer applications [22]. In contrast, 3D metal AM is limited to a few steels, aluminum and titanium alloys [25], and the maximum thermal conductivity is less than $200 \mathrm{~W} /(\mathrm{m} \cdot \mathrm{K})$. Although significant development in the metal AM has been made recently and part densities above $99 \%$ can be achieved by optimizing the process parameters [26], the reduction in heat transfer properties of AM parts is still a concern and more studies are needed, as a low porosity can reduce the effective thermal conductivity significantly. For porous media, a porosity of 0.1 could lead to about $20 \%$ reduction in the effective thermal conductivity [27] and another study showed the decrease is about 30\% [28]. In addition, there are not enough studies unveiling the change in thermal properties of metals fabricated by the 3D AM technology.In comparison, the porosity of IC parts can be precisely controlled depending on the material and process, making it suitable to fabricate fully dense metal products. Another practical concern is post-processing. There may be some unavoidable supporting structures left after the DMLS process and removing them can be difficult. In the IC process, the resin supporting structures from the SLA process are removed more easily before casting, which simplifies the post processing and increases the accuracy of the final part. All these characteristics make SLA-assisted IC a promising technique to manufacture complicated TO designs in metals. Some drawbacks of IC manufacturing include possible porosity of the final cast part, limitations in the minimum feature size and cost.

This paper applies a known TO solver to design passive heat sinks for natural convection applications and validates them against reference pin-fin heat sinks by both experiment and simulation. The heat sinks are optimized using a gradient based TO for a constant volume constraint and the pin-fin heat sinks are designed with the same volume as the TO designs. The results give a possible path to manufacturing complex TO designs for thermal systems, where high thermal conductivity is necessary. SLA assisted IC is also a more flexible approach to experimentally validating TO designs because more materials are available to the IC process than AM techniques for metals. This paper is structured as follows: Section 2 introduces the TO solver and summarizes the topological designs. For the comparison, five reference pin-fin heat sinks are designed using the same design domain and metal volume. Section 3 proposes and applies the SLA-assisted IC process to fabricate six heat sinks; Section 4 describes the natural convection test apparatus and the validation model based on COMSOL Multiphysics; Section 5 presents the experimental and simulation results, and compares TO designs with reference heat sinks, and Section 6 gives brief conclusions. 
184

185

186

187

188

189

190

191

192

193

194

195

196

197

198

199

200

201

202

203

204

205

206

207

208

209

210

211

212

213

214

215

216

217

218

219

220

221

222

223

224

225

226

\section{Topology optimization of heat sinks}

Topology optimization [1] is an iterative design process of distributing a specified volume of material in a given domain by optimizing an objective function and fulfilling a set of constraints. Initially developed for structural mechanics, nowadays the method is applied in a wide range of disciplines like photonics and electromagnetics, fluid mechanics, heat transfer, etc. The design problem considered in this article is the optimization of heat sinks for LED lamps [19, 20,38] where the goal is to distribute conductive material by minimizing the volumetric thermal compliance of the system. This is equivalent to minimizing the average temperature of the heat source. A density-based formulation is used, initially presented in $[18,19]$. Details of the specific problem setup are given in [20,38]. For brevity, only the most important parts are included in the presentation here. A heater stacked between an aluminum plate and an insulation foam layer form the non-design heat supply part of the optimization setup. A highly conductive material is distributed in a cylindrical design domain attached to the heat source. The numerical modeling of the physical response is based on the Navier-Stokes and convection-diffusion equations where the Boussinesq approximation is introduced to take density-differences due to temperature-variations into account.

$$
\begin{gathered}
\rho_{0} u_{j} \frac{\partial u_{i}}{\partial x_{j}}-\mu \frac{\partial}{\partial x_{j}}\left(\frac{\partial u_{i}}{\partial x_{j}}+\frac{\partial u_{j}}{\partial x_{i}}\right)+\frac{\partial p}{\partial x_{i}}=-\alpha_{B}(x) u_{i}-\rho_{0} \beta\left(T-T_{0}\right) g_{i} \\
\frac{\partial u_{j}}{\partial x_{j}}=0 \\
\rho_{0} c_{p} \frac{\partial T}{\partial x_{j}}-\frac{\partial}{\partial x_{j}}\left(k(x) \frac{\partial T}{\partial x_{j}}\right)=q^{\prime \prime \prime}(x)
\end{gathered}
$$

where $\alpha_{B}(x)$ is the spatially-varying Brinkman penalization coefficient taking values 0 for fluid and $\infty$ for solid, $k(x)$ is the spatially-varying thermal conductivity and $q^{\prime \prime \prime}(x)$ is a volumetric heat source term. The equations are defined in the domain $\Omega=\Omega_{s} \cup \Omega_{f}$, where $\Omega_{s}$ denotes the part of the domain occupied by solid and $\Omega_{f}$ the part occupied by fluid. The penalization, $\alpha_{B}(x)$, ensures zero velocities in the solid, however, for numerical simulations the penalization is relaxed and $\alpha_{B}$ takes finite value in the part occupied with solid [18]. Similar to the Brinkman penalization, the conductive coefficient takes the value $k_{f}$ in the fluid and $k_{s}$ in the solid. The volumetric heat source $q^{\prime \prime \prime}(x)$ is only active within a predefined domain coinciding with the actual heat source [38]. The LED lamp is modelled as freely suspended in open space, with a varying design orientation. Figure 1 shows the problem setup for the optimisation process. It shows the various subdomains and the boundary conditions of the simplified model, along with the orientation angle, $\alpha$. For further details and exact dimensions, see Ref. [38].

The PDEs (1)-(3) are discretized using the finite element method presented in [18, 19], on a regular grid occupying a parallelepiped with dimensions $150 \mathrm{~mm} \times 150 \mathrm{~mm} \times 250 \mathrm{~mm}$ including a $1 \mathrm{~W}$ heat source. The gradient-based optimization process is iterative and requires a new state as well as an adjoint solution for each design update. A density field $\gamma(x)$ is utilized to represent the design. The field takes values between zero and one, where $\gamma=1$ represents the fluid and $\gamma=0$ represents the solid. With the help of the density, the Brinkman penalization, $\alpha_{B}$, and the conductivity coefficient, $k$, are interpolated between their values for solid and fluid. The gradients of the objective function are obtained by adjoint sensitivity analysis as described in $[17,18]$. 


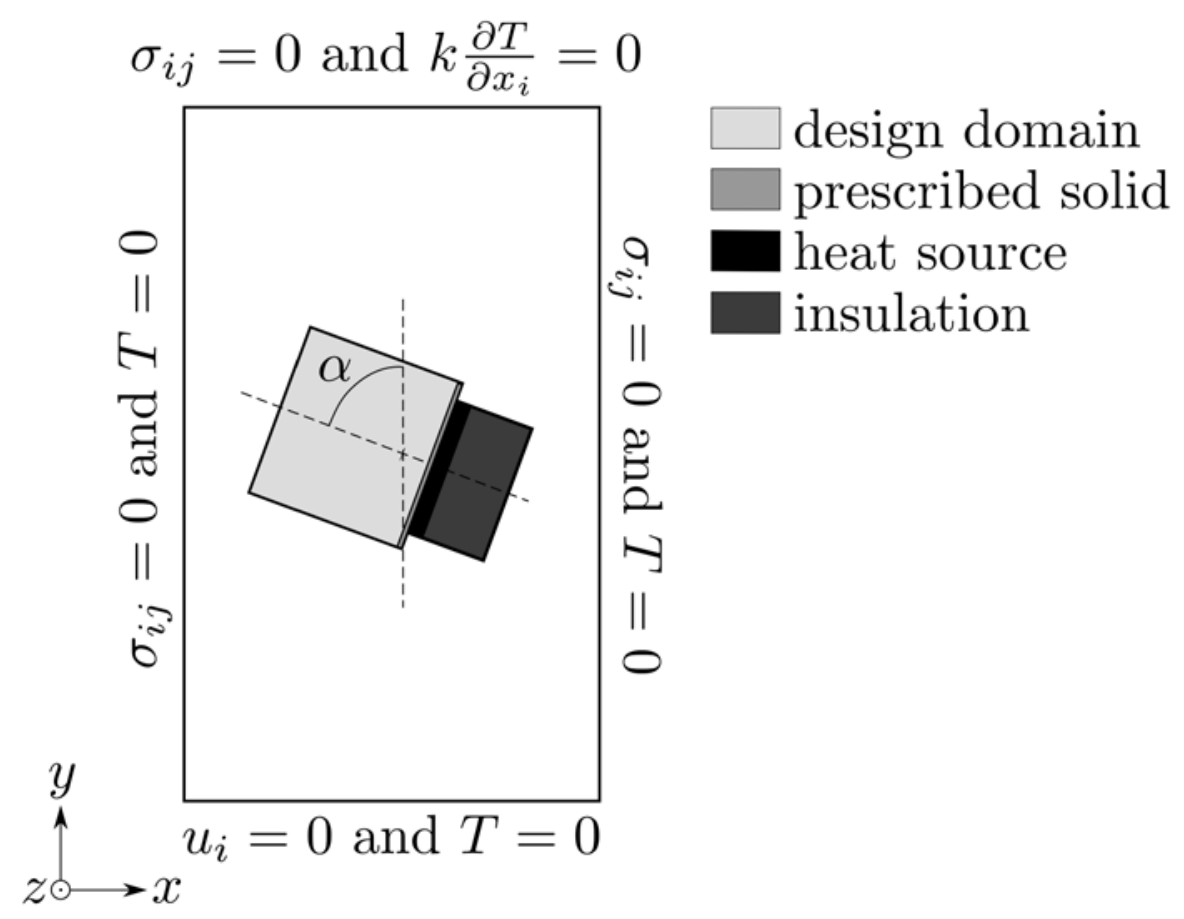

Figure 1. Illustration of the problem setup for the optimisation process. The various domains and boundary conditions are shown. Please see Ref. [38] for further details and exact dimensions.

The optimization problem is regularized using PDE filtering [29, 30] to ensure mesh-independent designs and control of minimum length scale. The computational time is controlled by parallelizing the state solution process and the design updates [33]. For the results presented here runtimes vary from a day on 2000-4000 CPUs to a week on smaller clusters. The density distribution is updated in parallel [34] using the Method of Moving Asymptotes (MMA) [35].

Figure 2 shows three heat sinks designed by TO with different boundary conditions. The first two heat sinks in Figure 2 (a) and (b) are optimized when the heating surface is perpendicular to the horizontal direction, and they are named "horiz1" and "horiz2", respectively. The optimization domains are chosen as $1 / 4$ and $1 / 8$ of the full size domain for the two designs respectively. Based on this constraint, "horiz1" is bilateral symmetric, while "horiz2" has an 8-fold mirror symmetry geometry. For the heat sink "horiz1", two wide thin structures extend from the base and bend toward the center near the boundary of the design space, separating the air flow into three streams. Two tree-like structures are located at the top and bottom positions from the side view, and they fully contact the main flow stream in the middle. The cross sections of these four branches are streamlined in the flow direction, which helps reducing the flow resistance. Many smaller sub-branches are generated on the crown of the four main trunks, which could dissipate heat in an efficient way. The heat sink "horiz2" consist of two sets of 4 branches: one set of tall branches with elongated cross-sections to guide the flow in and out of the heat sink; and one set of shorter tree-like branches utilising the central flow as for "horiz1". The performance of "horiz2" is expected to be below that of "horiz1" due to the reduced design freedom imposed by the additional symmetry requirements. Due to the required repetition, the branches cannot be designed as would be optimal, but rather optimal in an averaged sense with respect to the rotation of the design. A more detailed analysis of the design features and their effect can be found in Reference [38] for similar heat sink designs in aluminium 
256

257

258

259

260

261

262

263

264

265

266

267

268

269

270

271

272

273

274

275

276

277

278

279

280

281

282

283

284

285

286

287

288
In addition, the third heat sink "vert" in Figure 2 (c) is designed for orientation in the vertical direction and the heating surface is perpendicular to the gravitational direction. The optimization domain is $1 / 8$ of the full domain, which generates an 8-fold symmetric design. As seen in Figure 2 (c), there are many tiny branches growing from the eight main trunks, and the upper sections of these branches are connected to each other, forming a thin barrel structure, yielding a chimney-effect that increases the mass flow into the heat sink. In the middle of "vert", eight small arrow-like branches extend from the base of the main trunks, which help dissipating heat in the center of the heat sink. All the TO heat sinks have a base plate with a thickness of approximately $3 \mathrm{~mm}$.

Five pin-fin heat sinks as seen in Figure 3 are also designed for comparison in both experiment and simulation validations. To allow for a reasonable comparison, the overall size is held the same as the TO designs, which has a diameter $D$ of $65 \mathrm{~mm}$ and a height $H$ of $60 \mathrm{~mm}$. All reference heat sinks have 3 $\mathrm{mm}$ thick base plates, which are the same as for the TO heat sinks. In addition, the metal volume $V_{m}$ for each reference heat sink is controlled to be close to $48.2 \mathrm{~cm}^{3}$, which is the average volume of the TO designs. A variation of less than $2.5 \%$ in the volume exists due to the choice of the pin diameter and pin distribution. The void fraction, or so-called porosity, is defined as $\varepsilon=1-4 V_{m} / \pi D^{2} H$, which is about 0.76 for each heat sink. The pins are $57 \mathrm{~mm}$ long for each pin-fin heat sink, giving a total heat sink height of $60 \mathrm{~mm}$, and the pin diameter ranges from 2.7 to $8.7 \mathrm{~mm}$ with a step of $1.5 \mathrm{~mm}$, corresponding to a length-to-diameter ratio from 22.2 to 6.9. The details of the heat sinks are listed in Table 1. In general, the pins are distributed evenly. The fin number and fin distance are determined by the constrained volume and porosity. Some outer pins are cut smaller to fit the profile of the design space. Although many studies, i.e. Ref. [31, 32], have been implemented to optimize the pin diameter, lengthto-diameter ratio and the porosity, this paper is not intended to optimize all the parameters and only the pin diameter varies for comparison. The pin size was chosen based on modelling of the heat sink and in that way we perform an experimental size optimization of the pin fin heat sinks as a comparison to the TO designs. Additional optimization of the pin fin heat exchangers could give an improvement in performance and thus possibly reduce the advantage of the TO designs. The TO heat sinks have a similar surface area of about $261-283 \mathrm{~cm}^{2}$ as seen in Table 1, while the surface areas of the reference heat sinks range from 255 to $677 \mathrm{~cm}^{2}$ depending on the pin diameters. Even though the surface areas of the TO heat sinks are lower than that of most of the reference groups, it is anticipated that TO designs will give superior performance, as the optimized flow management contributes significantly to the heat dissipation. 

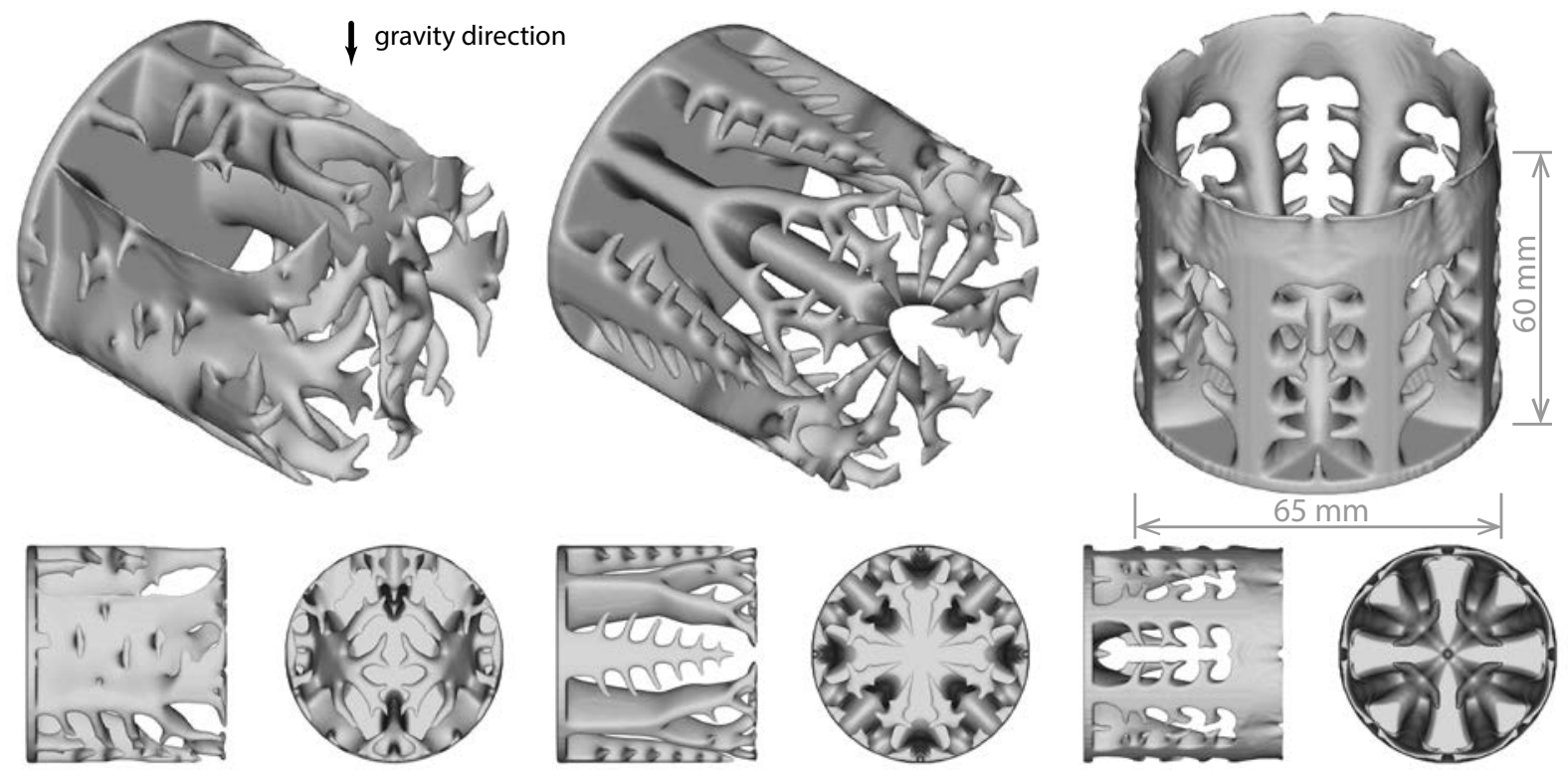

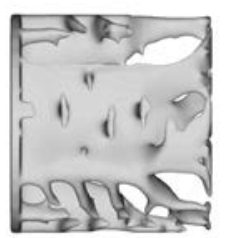

(a) TO heat sink for horizontal position based on $1 / 2$ domain (horiz1)

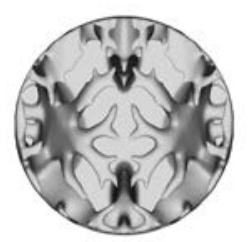

(b) $\mathrm{TO}$
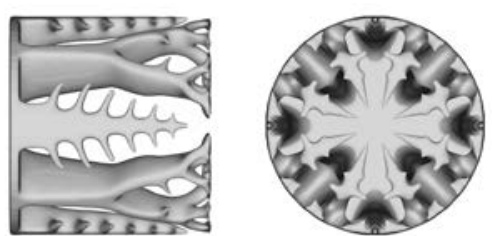

heat sink for horizontal position based on 1/4 domain (horiz2) (c) TO heat sink for vertical position based on 1/4 domain (vert)
289

291

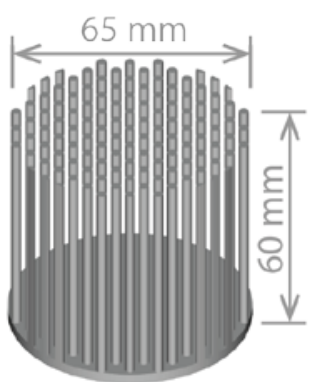

(a) Pin-fin heat sink with

\begin{abstract}
$D_{\mathrm{p}}=2.7 \mathrm{~mm}(\mathrm{pf}-2.7 \mathrm{~mm})$
\end{abstract}
Figure 2. Heat sinks designed by topology optimization

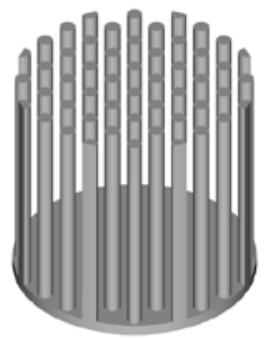

(b) Pin-fin heat sink with $D \mathrm{p}=4.2 \mathrm{~mm}$ (pf-4.2mm)

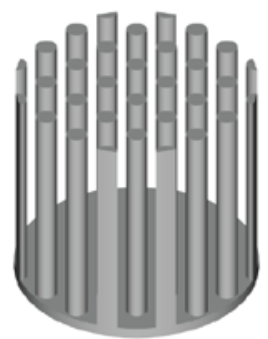

(c) Pin-fin heat sink with $D \mathrm{p}=5.7 \mathrm{~mm}$ (pf-5.7mm)

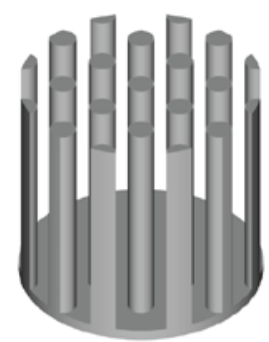

(d) Pin-fin heat sink with $D_{\mathrm{p}}=7.2 \mathrm{~mm}$ (pf-7.2mm)

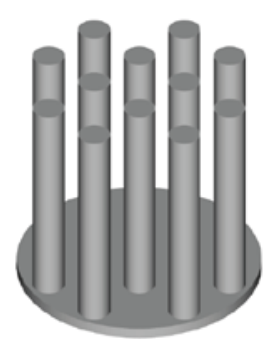

(e) Pin-fin heat sink with $D \mathrm{p}=8.7 \mathrm{~mm}(\mathrm{pf}-8.7 \mathrm{~mm})$

Figure 3. Reference pin-fin heat sinks

Table 1. Characteristics of TO and reference heat sinks

\begin{tabular}{|c|c|c|c|c|c|c|c|c|}
\hline & "horiz1" & "horiz2" & “vert” & $\begin{array}{c}\text { "pf- } \\
2.7 \mathrm{~mm} "\end{array}$ & $\begin{array}{c}\text { "pf- } \\
4.2 \mathrm{~mm} "\end{array}$ & $\begin{array}{c}\text { "pf- } \\
5.7 \mathrm{~mm} \text { " }\end{array}$ & $\begin{array}{c}\text { "pf- } \\
7.2 \mathrm{~mm} 2\end{array}$ & $\begin{array}{c}\text { "pf- } \\
\text { 8.7mm" }\end{array}$ \\
\hline Design volume $\left[\mathrm{cm}^{3}\right]$ & 48.3 & 47.7 & 48.6 & 47.6 & 48.8 & 48.8 & 49.4 & 48.7 \\
\hline Design mass* $[\mathrm{g}]$ & 362.3 & 357.8 & 364.5 & 357.0 & 366.0 & 366.0 & 370.5 & 365.3 \\
\hline Design surface area $\left[\mathrm{cm}^{2}\right]$ & 265.9 & 261.4 & 283.4 & 677.5 & 456.2 & 456.2 & 378.5 & 255.1 \\
\hline Design pin number [-] & - & - & - & 121 & 52 & 32 & 21 & 12 \\
\hline Measured mass [g] & 364 & 367 & 385 & - & 366 & 371 & 358 & - \\
\hline
\end{tabular}

$*$ Calculated based on the design volume and an estimated density of $7.5 \mathrm{~g} / \mathrm{cm}^{3}$

\section{Fabrication of TO heat sinks using SLA-assisted investment casting}


The process of investment casting used in this study is derived from jewelry fabrication, which is suitable for fast prototyping. It consists of the five steps as seen in Figure 4. The process starts with the 3D SLA printing of the castable resin pattern in step (a), which is an alternative to the traditional wax injection molding or manual sculpting. In the molding process, the resin part that constitutes the heat sink pattern is glued to a wax gating system that controls the metal flow into the mold. The whole pattern is then fixed inside a steel flask. A plaster slurry is poured into the flask to form the mold in step (b) and it is degassed to remove bubbles before hardening. In step (c), the mold is placed in a furnace and the temperature is increased in steps for melting and burning out the castable resin and wax. The mold is then slowly cooled, and when it reaches the required casting temperature, the molten metal is poured into the cavity through the gating system, forming the desired metal part in step (d). Finally, the metal part is demolded after cooling down (step (e)) and it is post-processed, including removing the gating structures, cleaning and polishing, etc.

We design and cast the heat sinks based on Britannia metal (Sn-Sb-Cu alloy), which is a tin-based alloy consisting of $92 \%$ tin, $6 \%$ antimony and $2 \%$ copper. The thermal diffusivity of Britannia metal is measured using a Netzsch 457 Laser Flash Apparatus, which is about $27.3 \mathrm{~mm} / \mathrm{s}$ at room temperature. The thermal conductivity is estimated as $47 \mathrm{~W} /(\mathrm{m} \cdot \mathrm{K})$ using an estimated density of $7500 \mathrm{~kg} / \mathrm{m}^{3}$ and heat capacity of $230 \mathrm{~J} /(\mathrm{kg} \cdot \mathrm{K})$. It has a low casting temperature of about $260{ }^{\circ} \mathrm{C}$ and it is relatively easy to avoid casting voids that can increase the porosity of the finished part due to its low shrink rate and low pour temperature compared to most metals.

Figure 5 (a) and (b) show the TO design "horiz2" and the resin pattern printed by a Formlabs SLA printer, i.e. FORM2. To avoid deformation in the casting procedure, two rings with guiding channels are added onto the top of the heat sink branches. Without these constrained rings, the branches tended to contract toward the center and the thin base plate bent in a preliminary test. The finished plaster mold is presented in Figure 5 (c), and there is a spout in the middle to guide the liquid metal flow. After casting, the TO heat sink in Figure 5 (d) shows a yellow/golden appearance and all the details from the TO design are captured, although small inclusions on the surface of most of the heat sinks are present. The sprue structure is removed and the bottom plate is milled flat. In total, three TO and three reference heat sinks are fabricated as shown in Figure 6. A detailed view of the surface of a finished cast part is shown in Figure 7. The surface finish can vary depending on the location, but the two images in Figure 7 are representative of the general surface finish. The heat sinks are partly polished to remove any plaster or slag from the casting process, resulting in a silvery surface. All heat sinks were coated with graphite spray for IR imaging, and they are shown painted in Figure 6. The fabricated heat sinks show that SLA assisted IC has a high accuracy, making this technique suitable for prototyping TO designs. The mass of the fabricated heat sinks is measured and listed in Table 1 . The mass of the heat sink "vert" is about $4 \%$ larger than the prediction due to uncertainty in the casting and final milling processes, and the others have similar mass with a variation smaller than $3 \%$. Due to the complexity of the geometry it was not measured how the final geometry varied from the design and it is therefore impossible to predict how this uncertainty affects the final performance. 


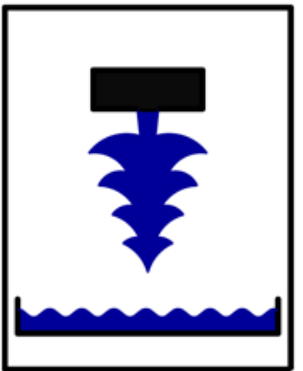

(a) 3D SLA printing of resin pattern 344

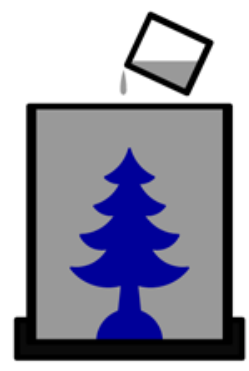

(b) Molding with plaster slurry

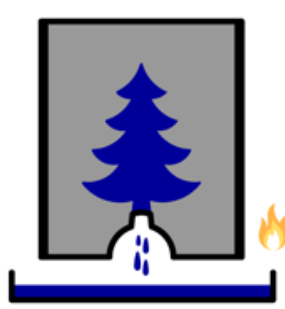

(c) Burning resin pattern out

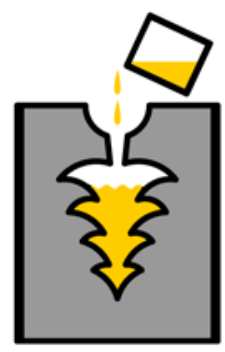

(d) Casting with molten metal

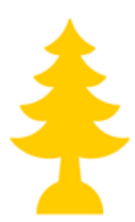

(e) Demolding and post-processing

Figure 4. Process of SLA assisted investment casting

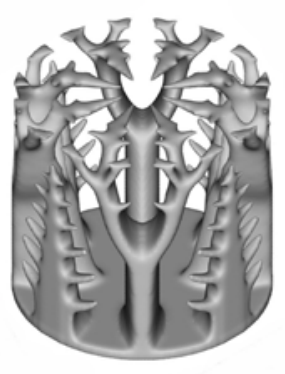

(a) TO design

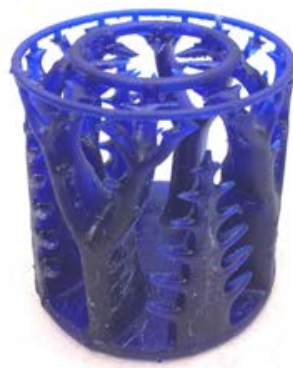

(b) Resin pattern

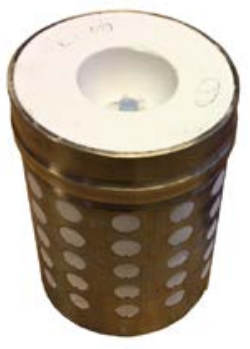

(c) Plaster mold

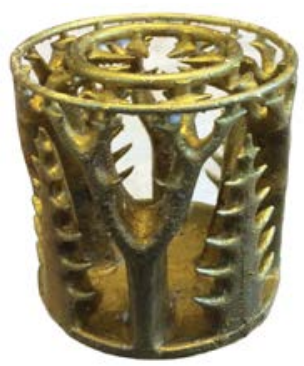

(d) Metal heat sink Figure 5. TO design of "horiz2", resin pattern by SLA printing, plaster mold, and heat sink in the Britannia metal (Sn-Sb-Cu alloy) before gates are removed. 


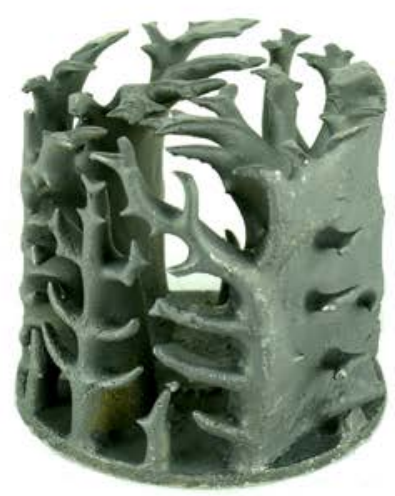

a) horiz1

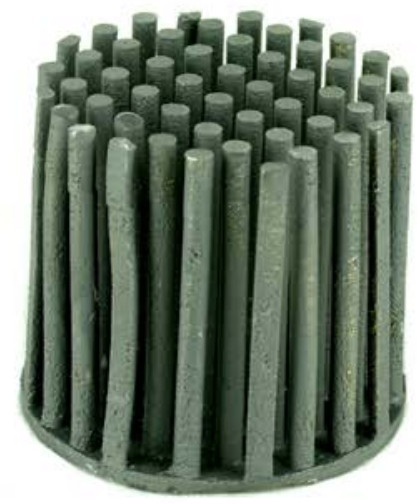

d) pf-4.2 mm

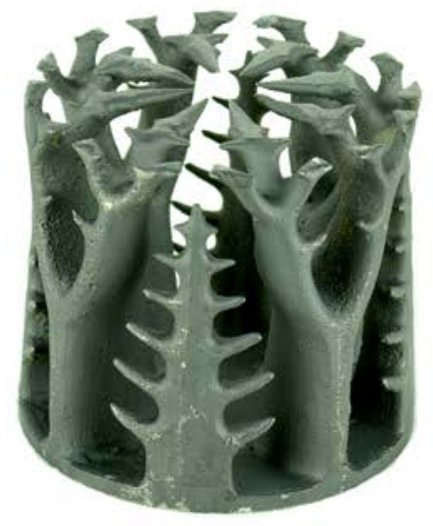

b) horiz2

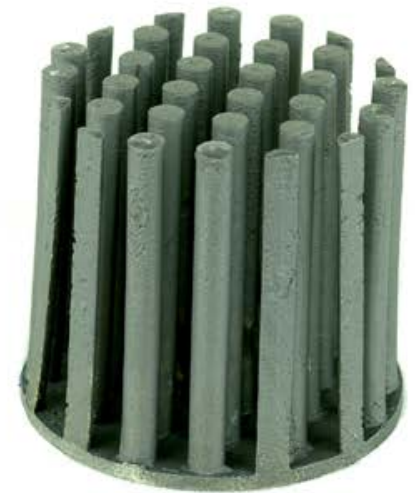

e) pf-5.7 mm

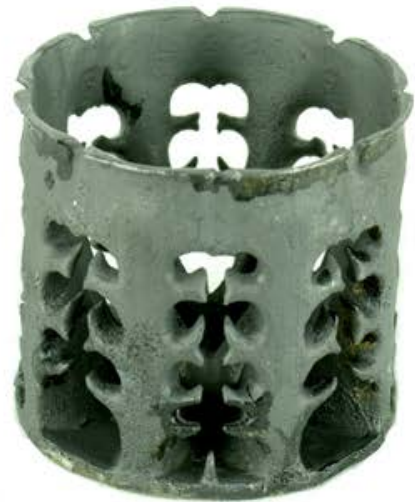

c) vert

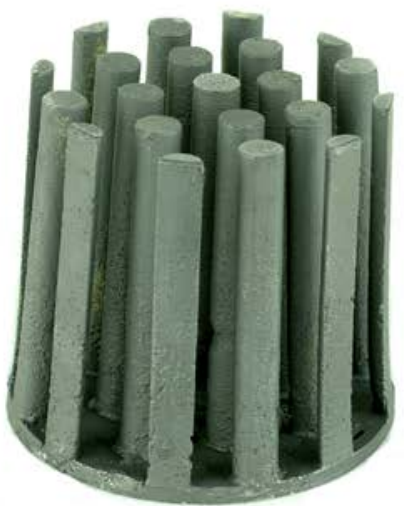

f) pf-7.2 $\mathrm{mm}$

Figure 6. TO and reference heat sinks fabricated by SLA-assisted investment casting. All heat sinks are coated with graphite paint for IR measurements.

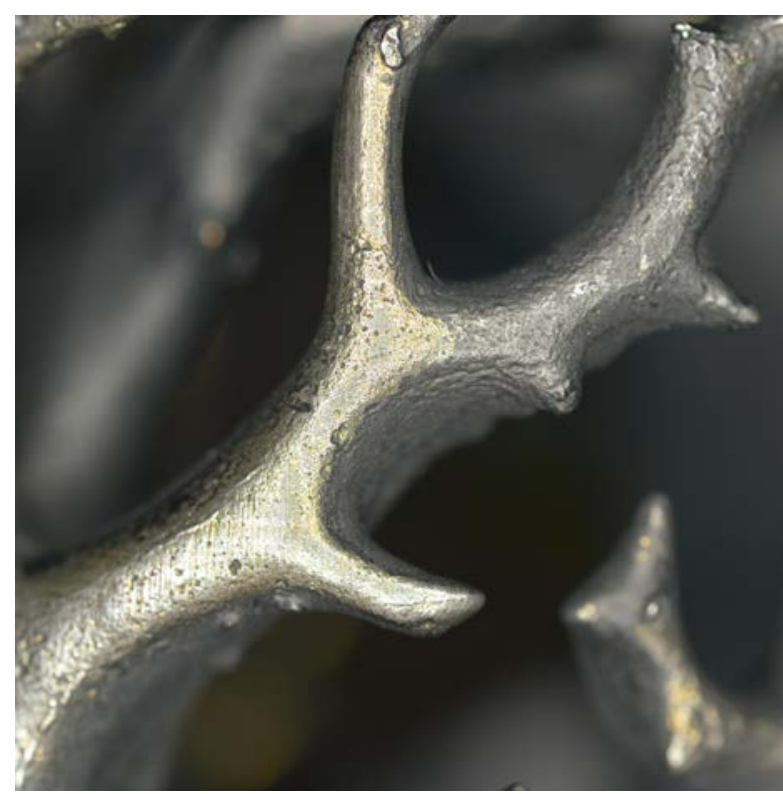

(a)

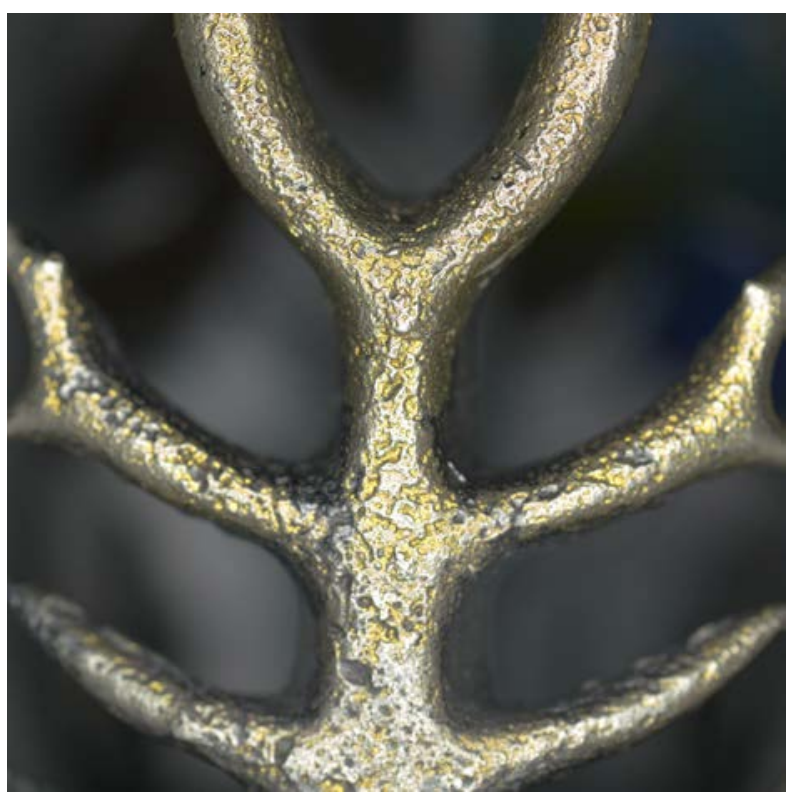

(b)

Figure 7. Detail of surface texture of "horiz1" cast part, (a) and (b). The size of the image is approximately $24 \mathrm{~mm}$ x $24 \mathrm{~mm}$ for each image. 
X-ray micro-tomography was performed to investigate the quality of the cast heat sink. In order to detect the presence of porosity at different length scales, two different tomographic scans were performed. Figure 8(a) shows the results of a large field of view scan, obtained using the lab micro-CT instrument Nikon XT H 225 (Nikon Metrology Ink.), at $200 \mathrm{keV}$ and 15 W. In order to improve transmission, an Sn filter of $1 \mathrm{~mm}$ thickness was used. The entire "horiz2" sample was scanned with a voxel size of 76.4 $\mu \mathrm{m}$. From the tomography results, the sample appears to be dense and no porosity could be detected at this scale. Figure 8(b) shows the results of a high-resolution scan, performed using the lab instrument Xradia Versa 420 (Carl Zeiss Inc.), at $150 \mathrm{keV}$ and $10 \mathrm{~W}$. The sample was obtained from a single pin from pf-4.2 mm that was turned down to a diameter of $2 \mathrm{~mm}$ and then analyzed with a voxel size of 2.2 $\mu \mathrm{m}$. In this case, some porosity was detected (red regions in Figure 8(b)). The pores were segmented from the rest of volume using interactive thresholding in Avizo (Visualization Sciences Group, FEI Company). From the segmented volume, the pore phase fraction was estimated to be less than $0.1 \%$, showing that the casting quality is adequate and is not expected to affect the experimental results.
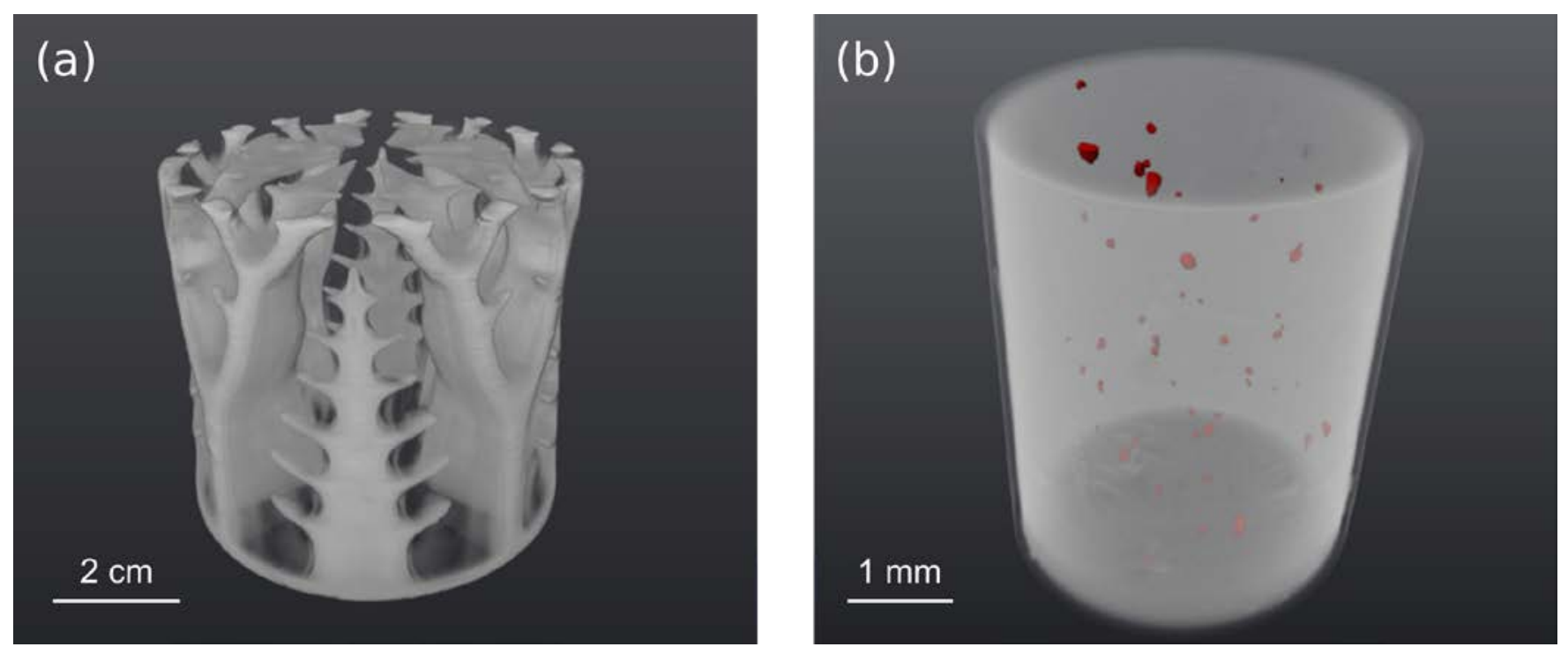

Figure 8. Volume renderings from the X-ray tomographic data of (a) "horiz2” and (b) sample obtained from a single pin of "pf-4.2 mm”. In (b), the detected porosity is highlighted in red.

\section{Experimental apparatus and validation simulation model}

This section introduces the methodology for validating the TO heat sinks based on both experiment and simulation. In both cases, the heat sinks are set in an open environment for natural convection tests to emulate the TO model. A heat source is applied on the aluminum heating plate attached to the bottom of the heat sinks to impel the natural convection flow. The bottom of the heating plate and the heat source are insulated and a vast majority of the heat is conducted through the conductive metal and then to the environment by convection. Based on this concept, a natural convection test apparatus is constructed and a validation simulation model based on COMSOL Multiphysics is built as described below.

\subsection{Experimental setup}

As seen in Figure 9, the test apparatus consists of two main parts: the outer shield and the test section supported by a tripod. The outer shield is a round cylinder with an open top and bottom with a diameter of about $1 \mathrm{~m}$, which is about 15 times larger than the heat sink diameter. A preliminary experimental test 
shows the outer shield can effectively reduce flow interruptions from other laboratory equipment and human activity. It is also noted that the test results do not change with or without the outer shield, if the open environment is held calm and stable. Still, the outer shield is used to reduce the interruption to the lowest level. Another reason for using the shield is that it can largely reduce reflections disturbing infrared thermal imaging conducted in the test. As seen in Figure 9 (a), a $200 \mathrm{~mm}$ long rod is made of fiberglass reinforced plastic and fixed to the tripod for supporting the test section, which is also used to reduce the flow disruption by the tripod. The orientation and position of the heat sink are varied by adjusting the tripod platform. From bottom to top, a thin plastic plate, an Omega KHLV 101-10 thin film heater, a $52 \mathrm{~mm}$ round alumimun plate and the heat sink are mounted together by two steel screws, forming the heating section for the test. Thermal paste, Omega OT 201, which has a thermal conductivity of about $2.2 \mathrm{~W} /(\mathrm{m} \cdot \mathrm{K})$, is applied to the surface between the heater and the alumimun plate, as well as the surface between the heat sink base plate and the alumimum plate. In addition, a small amount of the thermal paste is applied on the screw thread to improve the overall conductivity of the screw connection. The screws are tightened firmly to ensure the contacting pressure and then to reduce the thermal resistance. The heat sink base is machined, as well as the aluminum plate, which should result in a good thermal interface as suggested by [39]. The heater is permanently attached to the aluminum plate and only the heat sink is switched when changing the sample. Therefore, the contacting condition and the thermal resistance are estimated as similar from test to test. The thin plastic plate is a thermal insulator and it is viewed as a part of the insulation foam in the schematic Figure 9 (b) and also the simulation model presented in the next section. The whole heating section is installed on the insulation housing based on a four-point supporting structure using small nylon screws. There is a gap between the heat section and the base, forming an air insulation to reduce the heat loss. The insulation housing is made of fiberglass reinforced plastic and Armaflex insulation foam is filled inside. The insulation housing and the insulation foam have a thermal conductivity of about 0.5 and $0.037 \mathrm{~W} /(\mathrm{m} \cdot \mathrm{K})$, respectively.

Resistance temperature detectors (RTDs) PT100 from Measurement Specialties, Inc. are used to acquire the temperature on the aluminum heating plate and the heat sink surface, as seen in Figure 9. These micro RTD sensors have a dimension of $2 \times 2 \mathrm{~mm}$ and the measurement error is $\pm 0.3^{\circ} \mathrm{C}$. The temperature data is recorded by a National Instruments (NI) 9216 PT100 RTD module with a NI cDAQ 9181 acquisition device. The thin film heater is powered by a TTi EL302P power supply unit, which also logs heater power with accuracies of $0.1 \%$ in voltage and $0.3 \%$ in current. The overall uncertainty of the heater power is estimated as $0.4 \%$. Figure 9 (d) shows that an infrared FLIR SC5000 thermal imaging camera is set up for capturing the temperature distribution on the heat sink surface.

In the test, the power input $Q$ and the temperatures on the heating plate and the branches are measured. Heat is dissipated from the heat sink to the open environment by convection $\left(Q_{\text {conv }}\right)$, radiation $\left(Q_{\text {rad }}\right)$ and conduction through the insulation, which is also the loss $\left(Q_{\text {loss }}\right)$. Therefore, we have

$$
Q=I V=Q_{\mathrm{conv}}+Q_{\mathrm{rad}}+Q_{\mathrm{loss}}
$$

where $V$ and $I$ are the voltage and current applied to the thin film heater.

The convection term $Q_{\text {conv }}$ is:

$$
Q_{\text {conv }}=\int h_{\text {local }}\left(T_{\mathrm{m}}-T_{\mathrm{a}}\right) \mathrm{d} A_{\mathrm{m}}
$$


438

where $h_{\text {local }}, T_{\mathrm{m}}, T_{\mathrm{a}}$ and $A_{\mathrm{m}}$ are the local heat transfer coefficient, metal surface temperature, air temperature and surface area for convection.

Using the method presented in Ref. [36], the heat transfer by radiation between the heat sink and the imaginary air enclosure surface is:

$$
Q_{\mathrm{rad}}=\frac{A_{\mathrm{a}} \sigma\left(T_{\mathrm{m}}{ }^{4}-T_{\mathrm{a}}{ }^{4}\right)}{\frac{A_{\mathrm{a}}}{A_{\mathrm{m}}}\left(\frac{1-\epsilon_{\mathrm{m}}}{\epsilon_{\mathrm{m}} A_{\mathrm{m}}}\right)+1}
$$

where $\sigma, \epsilon_{\mathrm{m}}, A_{\mathrm{m}}$ and $A_{\mathrm{a}}$ are the Stefan-Boltzmann constant, emissivity of the metal surface, surface area of the heat sink and surface area of the imaginary air enclosure, respectively. The imaginary air enclosure here is the domain with a diameter of $65 \mathrm{~mm}$ and height of $60 \mathrm{~mm}$.

The loss is mainly the conduction through the insulation housing. Thus $Q_{\text {loss }}$ becomes:

$$
Q_{\text {loss }}=\int k_{\text {eff }} \frac{T_{\mathrm{m}}-T_{\mathrm{a}}}{x_{\text {cond }}} \mathrm{d} A
$$

where $k_{\text {eff }}, x_{\text {cond }}$ and $A$ are the effective thermal conductivity, distance and surface area for conduction.

In this study, $Q_{\text {rad }}$ and $Q_{\text {loss }}$ are estimated at about a total of $0.19 \mathrm{~W}\left(0.075 \mathrm{~W}\right.$ for $Q_{\text {rad }}$ and $0.115 \mathrm{~W}$ for $\left.Q_{\text {loss }}\right)$ out of the total $1.08 \mathrm{~W}$ of the experimental heating power. For different heat sinks, these ratios do not change much, as the temperature $T_{\mathrm{m}}$ is at the same level. Therefore, a direct way of evaluating the heat sinks is to compare the temperature at the heating plate, $T_{\mathrm{h}}$. Lower $T_{\mathrm{h}}$ represents lower thermal resistance which corresponds to better heat dissipation performance. In principle, the overall heat transfer coefficient and the dimensionless Nusselt number can be deduced from the measured surface temperatures. However, the temperature distribution on the TO heat sinks is unique and the measurement at different positions is difficult, due to the complicated geometry. Therefore, we mainly compare the temperatures at the heating plate and the overall heat resistance in the experiments. In addition, simulations could provide more details including the local heat transfer coefficient and the average heat flux, which will be discussed in Section 5. 


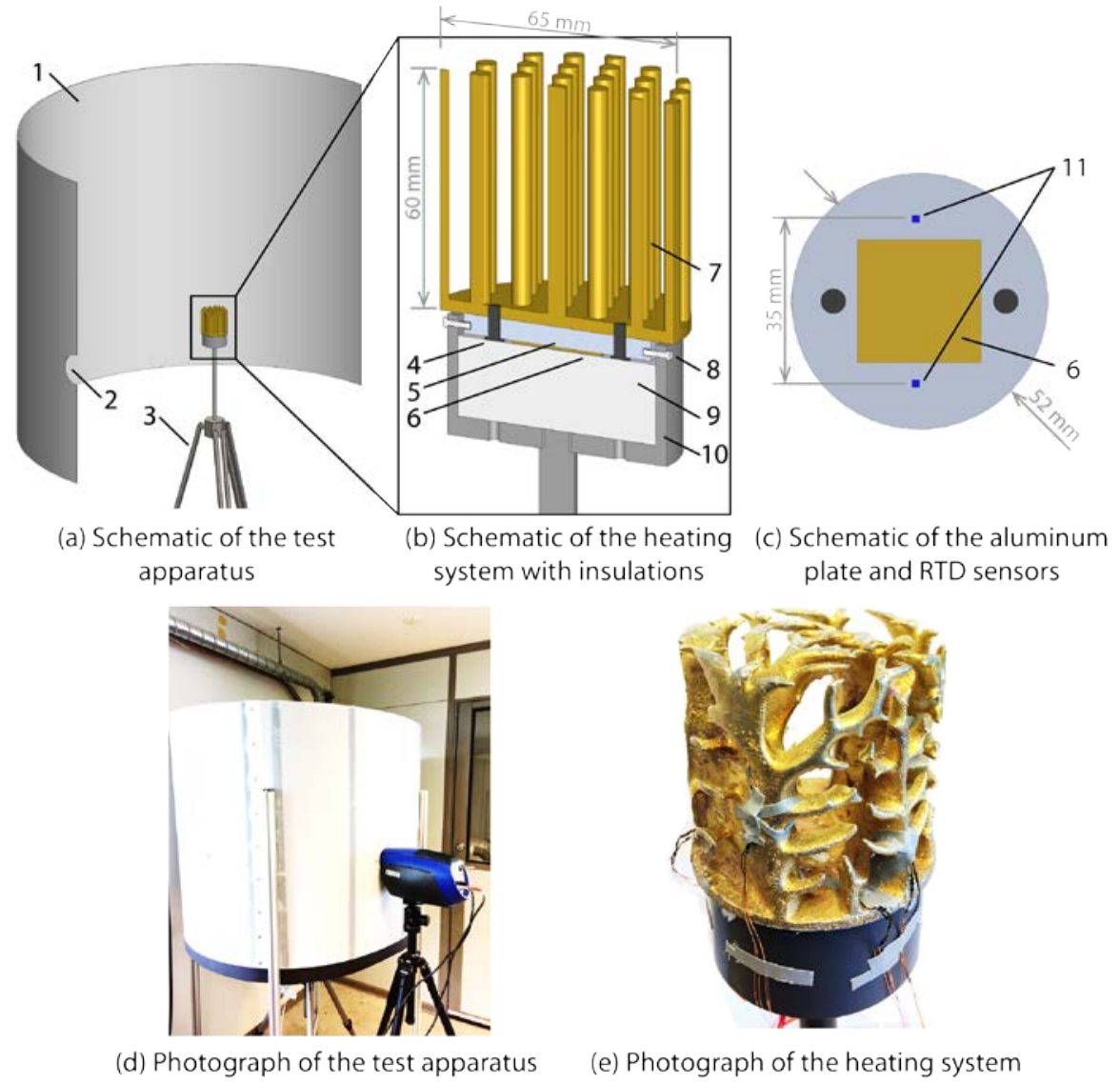

Figure 9. Schematic diagrams (a)-(c) and photographs (d)-(e) of the heat sink test apparatus (1, outer shield; 2: viewport for IR camera; 3: tripod; 4: steel screw; 5: aluminum heating plate; 6: thin film heater; 7: heat sink; 8: nylon screw; 9: insulation foam; 10: fiberglass housing; 11: RTD temperature sensors)

\subsection{Simulation model}

As mentioned above, 3D models of the analyzed heat sinks are implemented in COMSOL Multiphysics, as seen in Figure 10. The modeling in COMSOL performed with the same approach as described in section 2, which is to use the Boussinesq approximation for free convection. However, solid and fluid domains are modelled separately with explicit representation of the solid-fluid boundary in the validation model [37]. That is, we set the buoyancy force to $\rho_{\mathrm{a}} \beta\left(T-T_{0}\right) g$ in the $y$-direction, where $\rho_{\mathrm{a}}$ is the density of ambient air, $\beta$ is the coefficient of thermal expansion and $g$ is gravity. The air domain is separated into two subdomains, which are concentric cylinders of different heights: one is the inner domain surrounding the heat sink which is resolved with finer mesh; the other domain is further away from the heat sink and is resolved with a coarser mesh. The inner domain has a diameter and height of $200 \mathrm{~mm}$. The outer domain has a diameter of $600 \mathrm{~mm}$ and height of $800 \mathrm{~mm}$. The bottom of the inner domain is $200 \mathrm{~mm}$ above the bottom of the outer domain. The lateral surface of the outer air domain is set as an open boundary; the bottom of both domains is set as an open boundary as well; the top is set as outflow boundary. The test section is set inside the inner air domain. As in the experimental setup, the heater, aluminum plate and heat sink are mounted together and the heat section is insulated by the housing and foam inside. Two copper wires that lead to the heater are also considered as shown in Figure 10(b) and the air gap is simulated using a thin film boundary. In the simulations, all properties are assumed temperature-independent, and the values used are listed in Table 2. 
Table 2. Summary of material properties used in the simulation model

\begin{tabular}{cccc}
\hline Property & Value & Property & Value \\
\hline$c_{p, \text { air }}[\mathrm{J} /(\mathrm{kg} \mathrm{K})]$ & 1003 & $\mathrm{k}_{\text {insulation }}[\mathrm{W} / \mathrm{m} \mathrm{K}]$ & 0.04 \\
$c_{\mathrm{p} \text {, aluminum }}[\mathrm{J} /(\mathrm{kg} \mathrm{K})]$ & 903 & $\mathrm{k}_{\text {Brit }}[\mathrm{W} / \mathrm{m} \mathrm{K}]$ & 41.6 \\
$\mathrm{c}_{\mathrm{p} \text {, glassiber }}[\mathrm{J} /(\mathrm{kg} \mathrm{K})]$ & 1170 & $\mu_{\text {air }}[\mathrm{Pa} \mathrm{s}]$ & $1.86 \mathrm{E}-05$ \\
$\mathrm{c}_{\mathrm{p} \text {, insulation }}[\mathrm{J} /(\mathrm{kg} \mathrm{K})]$ & 1300 & $\rho_{\text {air }}\left[\mathrm{kg} / \mathrm{m}^{3}\right]$ & 1.2 \\
$c_{p, \text { Brit }}[\mathrm{J} /(\mathrm{kg} \mathrm{K})]$ & 210 & $\rho_{\text {aluminum }}\left[\mathrm{kg} / \mathrm{m}^{3}\right]$ & 2702 \\
$k_{\text {air }}[\mathrm{W} / \mathrm{m} \mathrm{K}]$ & 0.026 & $\rho_{\text {glassfiber }}\left[\mathrm{kg} / \mathrm{m}^{3}\right]$ & 1600 \\
$k_{\text {aluminum }}[\mathrm{W} / \mathrm{m} \mathrm{K}]$ & 175 & $\rho_{\text {insulation }}\left[\mathrm{kg} / \mathrm{m}^{3}\right]$ & 80 \\
$k_{\text {glassiber }}[\mathrm{W} / \mathrm{m} \mathrm{K}]$ & 0.26 & $\rho_{\text {Brit }}\left[\mathrm{kg} / \mathrm{m}^{3}\right]$ & 7000 \\
\hline
\end{tabular}

495

496

497

498

499

500

501

502

503

504

505

506

507

508

509

510

511

512

513

Tetrahedral elements with linear shape functions are used and the total number of elements is approximately $0.6-1.5 \times 10^{6}$ depending on the complexity of the heat sink geometry. Four different mesh densities have been applied to discretize the domain and the mesh resolution increases from the outer air domain to the inner heat sink. A mesh dependence study is carried out, showing that the predicted temperatures change insignificantly with further refinement. A transient solver is used and a time period of $3000 \mathrm{~s}$ is simulated, which allows the heat sink to reach a steady state. In this study, we only address the comparison study at the steady state. The thermal interface material (TIM) is not included in the general modelling of the heat sinks because the thickness of the interface layer is not known, although its influence is estimated for two of the cases below for assessing its influence. The locations of the TIM are shown in Figure 10(b).

As mentioned above, three TO heat sinks are designed based on two different orientations, and the unique geometry guides the flow in different ways. Therefore, we investigate the effect of the orientation on the heat sinks in both experiment and simulation, where the orientation angle $\alpha$ is defined as seen in Figure 10 (d). In addition, the heat sink "horiz1" has the two air foil flap-like branches and the flow pattern will be significantly affected by the rotation angle $\beta$ as seen in Figure 10 (e). Therefore, the impact of the rotation angle is also studied for "horiz1".

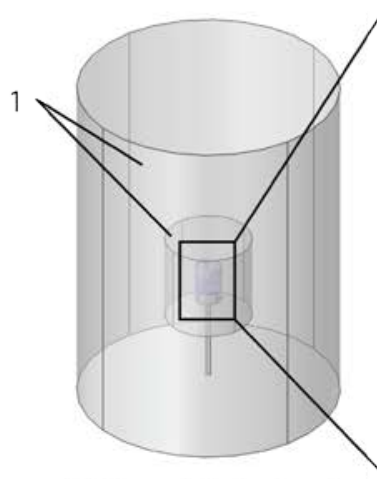

(a) Heat sink in the air domain

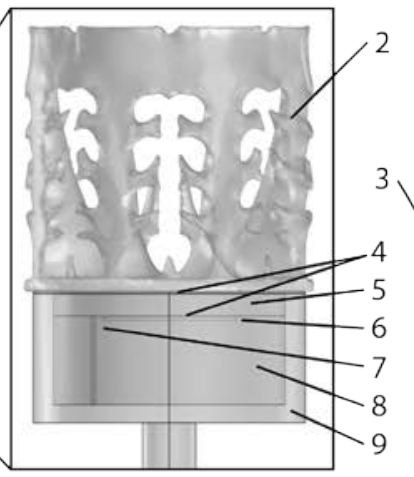

(b) Heating system

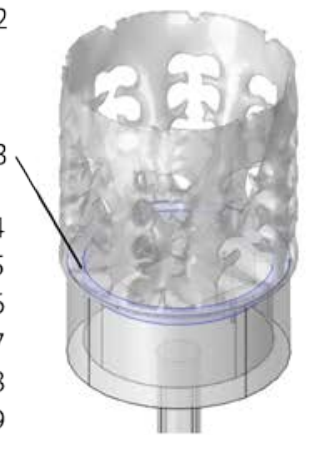

(c) Air gap

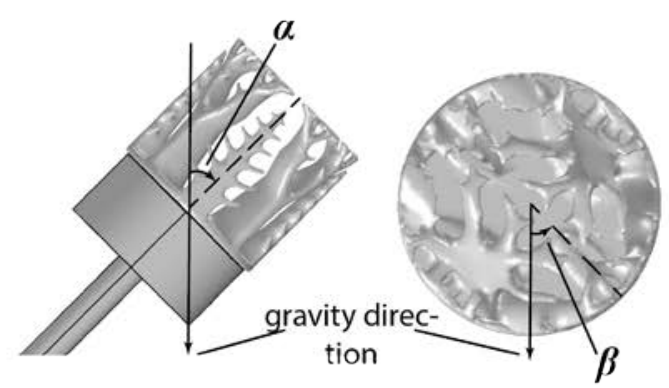

(d) Orientation angle

(e) Rotation angle
Figure 10. Heat sink validation model using the Boussinesq approximation based on COMSOL Multiphysics ${ }^{\circledR}$ (1: air domains; 2: heat sink; 3: air gap; 4: TIM (thin layer); 5: aluminum heating plate; 6: thin film heater; 7: copper cables; 8: insulation foam; 9: insulation housing)

\section{Results and discussion}


For each experimental test, a constant heating power is applied and the heat sink is heated to drive the natural convection flow. Until the heat sink reaches a steady state, the temperatures at the aluminum heating plate are recorded for comparison. It should be noted that measuring the heating plate rather than the heater itself removes the effects of the TIM between the heater and the plate. The temperature difference between two measured points on the heating plate as seen in Figure 9(c) is less than $0.3^{\circ} \mathrm{C}$, as the aluminum is highly conductive. Therefore, only the average value of the two measured temperatures is presented below. Simulations also predict small temperature variations across the aluminum plate, indicating that using the average of the measured temperatures is reasonable.

When comparing the predicted results to experiment, it is important to consider modelling uncertainties. For example, the temperature dependence of material properties has been ignored in the presented modelling. This assumption should hold when the heating power is relatively low, but at the highest heating powers, temperature spans above $25{ }^{\circ} \mathrm{C}$ were measured and some uncertainty of material properties is introduced. Experiments were conducted in a conventionally controlled indoor climate, which means that air temperatures can vary within the room and some air currents can occur. The variation in the air temperature distribution contributes to uncertainty in the boundary conditions applied in the COMSOL model and therefore can give significant uncertainty in prediction of free convection. Finally, radiation effects are difficult to accurately assess because the radiation properties of materials in the room are not always known. The range of materials that the heat sinks can interact with via radiation adds uncertainty to the heat transfer process.

\subsection{Comparison between TO and pin fin heat sinks}

\subsubsection{Temperature span for TO and pin fin heat sinks}

In total, all eight heat sinks listed in Table 1 are simulated and six are tested experimentally with two orientation angles $\alpha=0^{\circ}$ and $90^{\circ}$. The largest and smallest diameter pin-fin heat sinks listed in Table 1 were not fabricated because their predicted performance was lower than the others. Although the TO heat sinks are designed based on a heating power of $1.0 \mathrm{~W}$, higher heating power up to $7.9 \mathrm{~W}$ is applied for testing. Figure 11 presents the temperature spans, $\Delta T$, between the aluminum plate and the open air predicted by simulation and experimentally measured. The respective values are plotted in bars and dots. The air temperature $T_{\mathrm{a}}$ in the simulation is $16.5^{\circ} \mathrm{C}$ and it ranges from $15-23{ }^{\circ} \mathrm{C}$ in the measurements as they were conducted over several days. In each experimental test, $T_{\mathrm{a}}$ is stable. Multiple dots for the same heat sink represent repeated experiments. The dashed lines represent the average value of measured $\Delta T$ for "vert" at $\alpha=0^{\circ}$ and "horiz1" at $\alpha=90^{\circ}$, respectively, which are expected to be the best heat sinks for the two different orientation angles. The effect of the TIM on the results was calculated for the case with $1.1 \mathrm{~W}$ and $7.9 \mathrm{~W}$ heat load by modelling the TIM as a thin layer with a thickness of $100 \mu \mathrm{m}$ in the COMSOL model. In Ref [40], an LED cooling system was modelled with a $50 \mu \mathrm{m}$ TIM thickness so a $100 \mu \mathrm{m}$ thickness was used here as a conservative estimate. Including the effects of the TIM, the predicted temperature span increases by $3.3 \%$ for a $1.1 \mathrm{~W}$ heater power and $5.4 \%$ for a $7.9 \mathrm{~W}$ heater power.

The simulations in Figure 11 (a) show that "vert" realizes the lowest $\Delta T$ of about $6.1{ }^{\circ} \mathrm{C}$ compared to all other tested heat sinks. For the two TO heat sinks optimized for $\alpha=90^{\circ}$ ("horiz1" and "horiz2"), $\Delta T$ is significantly higher. Within the five reference heat sinks, the best performance is obtained with either "pf-4.2 mm" or "pf-5.7 mm" for different heating powers. Too small or too large pin diameters increase $\Delta T$ and the overall thermal resistance, which is defined as $\Delta T / Q$. The simulations show that too small 
568

569

570

571

572

573

574

575

576

577

578

579

580

581

582

583

584

585

586

587

588

589

590

591

592

593

594

595

596

597

598

599

600

601

602

603

604

605

606 pins with a dense distribution increase the flow resistance of "pf-2.7 mm”. Thus, the velocity between pins in the middle is much smaller than that for the other heat sinks, which also increases the temperature and forms a hot spot in the center of the heat sink. As a result, the overall heat resistance increases. For the heat sink "pf-8.7 mm" with large pins and a sparse pin distribution, the heat transfer area is largely reduced as seen in Table 1. Although the air flows freely through the pins, the thermal resistance of "pf-8.7 mm" is still high, reflected by the large temperature span. This indicates that the TO design "vert" guides the air flow in a better pattern with the same heat transfer area, realizing better heat transfer coefficient. With increasing heater power, the optimal pin diameter changes from 5.7 to 4.2 $\mathrm{mm}$, showing that smaller pins and a denser distribution are preferable for high heating powers. The simulations verify that "vert" performs best compared to the reference heat sinks when the heating power is $1.1 \mathrm{~W}$ or $3.0 \mathrm{~W}$. The performance of "vert" is slightly lower than the optimal reference design for $Q=5.0$ and $7.9 \mathrm{~W}$; however, the applied input power is much higher than the $1 \mathrm{~W}$ for which "vert" is optimized.

The experimental data shown in Figure 11 (a) further verifies that "vert" is a superior design within all the heat sinks when the heating power is $1.1 \mathrm{~W}$. The average $\Delta T$ of "vert" is also the lowest for higher heating powers, although the measured $\Delta T$ varies with a variation less than $4 \%$. In general, the experimental data follows the trends predicted by simulations well, and the average variation is within $8 \%$. Including the effect of the TIM increases the deviation to approximately $13.5 \%$. Possible reasons for the deviation may be the estimated radiation emissivity, material properties, fabrication variation, surface roughness and measurement error, etc.

The simulation and experimental results for $\alpha=90^{\circ}$ are presented in Figure 11 (b). In both simulations and experiments, "horiz1" is always the best design for different heating powers up to 5.0 W. For $Q=7.9$ $\mathrm{W}$, the modelled and measured $\Delta T$ of "horiz1" and "pf-5.7 mm" are similar, showing a similar heat dissipation performance. In contrast, "horiz2" exhibits a $\Delta T$ slightly higher than that of "horiz1", which is the second best. Although both "horiz1" and "horiz2" are designed by TO, "horiz1" is always better due to its unique design and larger freedom based on $1 / 2$ of the full domain as seen in Figure 6. Because "vert" was optimized for $\alpha=0^{\circ}$, it does not perform well with $\alpha=90^{\circ}$, neither in experiments nor simulations. This is the same for "horiz1" and "horiz2" with $\alpha=0^{\circ}$. This experimental cross validation shows that the TO heat sinks do give the best performance under the conditions for which they are optimized. For the reference heat sinks, the optimal design changes from "pf-7.2 mm" to "pf-5.7 mm" with increasing heating power, when the orientation angle is $90^{\circ}$.

It can be concluded from the presented data that the TO designs always perform better than the reference heat sinks in both experiments and simulations at the working conditions for which they are optimized. Their superior performance can also extend to higher values of heating power. The following section will present a detailed analysis of the TO and reference designs. 
(a) Orientation angle $\alpha=0^{\circ}$

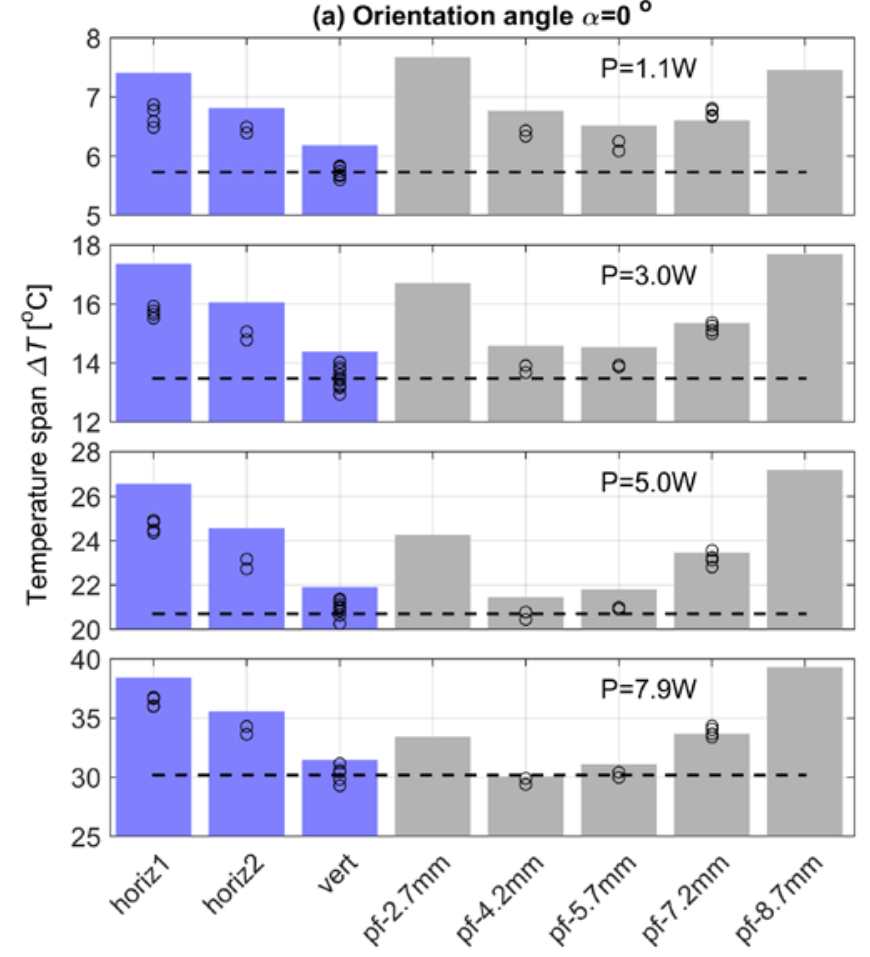

(b) Orientation angle $\alpha=90^{\circ}$

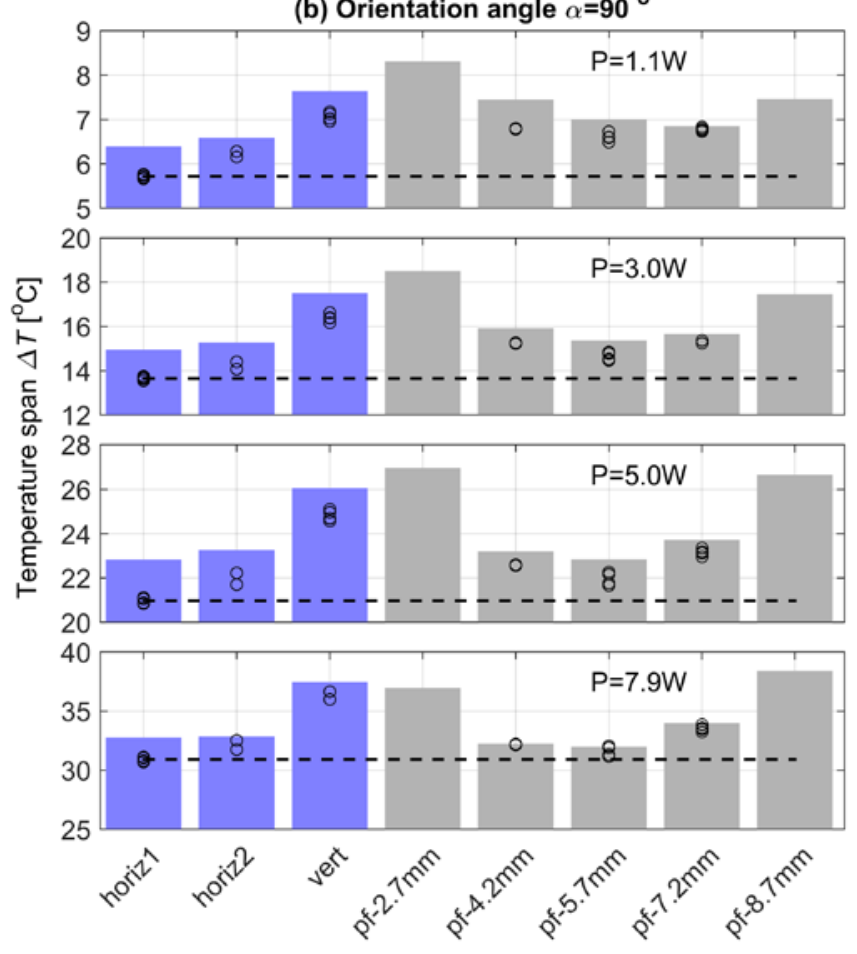

Figure 11. Temperature spans between the aluminum heating plate and the ambient for TO and pin fin heat sinks. Blue bars represent simulation results of TO heat sinks; grey bars represent simulation results of pin fin heat sinks; dots represent experimental results; a dashed line represents the average value of repeated experiments for "vert" with $\alpha=0^{\circ}$ or for "horiz1" with $\alpha=90^{\circ}$.

\subsubsection{Analysis of TO and pin fin heat sinks with $\alpha=0^{\circ}$}

For this analysis, we present the temperature distribution predicted by simulation for a $1 \mathrm{~W}$ power dissipation (corresponding to the conditions for optimization), experimentally measured temperature distribution, velocity field and local heat transfer coefficient predicted by simulation of "vert" and "pf5.7 mm" as seen in Figure 12 and Figure 13. The "pf-5.7 mm" is chosen as a benchmark because it gives the best pin fin performance at $1.1 \mathrm{~W}$. As the heat sink has a metallic and reflective surface, the reflection causes difficulties regarding capturing a good thermograph using the infrared thermal imaging camera. Therefore, we paint the heat sink using graphite spray. This unavoidably increases the emissivity to approach 1.0 which increases the percentage of heat dissipation by radiation. Therefore, the maximum $\Delta T$ presented in the following thermographs is lower than the simulation results and those results presented in Figure 11. Another reason for a lower $\Delta T$ is that the outer profile shown in the thermographs generally has a lower temperature than the inner. This effect caused by the graphite painting is studied in Section 5.3. Figure 12 (a) shows model prediction for the temperature of the heat sink surface and the air in the central cross sectional panel. The surface temperature exhibits a gradient from the base plate to the top ring structure. The air temperature distribution in the middle looks like a torch, showing that the air carrying the heat in the middle flows upward through the central part of the heat sink. The thermograph captured by the infrared camera in Figure 12 (b) shows a similar temperature distribution as the simulation. It also shows that the insulation performs as expected, based on the temperature profile of the support structure below the heat sink. The air from the open environment flows through the gaps between branches into the middle, exchanging heat with the metal as seen in Figure 12 (c). The hot air concentrates in the middle and flows upward, forming a similar 
636

637

638

639

640

641

642

643

644

645

torch-shape distribution as the air temperature. Figure 12 (d) presents the local heat transfer coefficient at the heat sink surface, which is $\delta Q_{\text {conv }} /(\Delta T \delta A)$ at a local position. It is seen that the local heat transfer coefficient of the outer surface is higher than that at the inner surface, and the highest $h_{\text {local }}$ is found on the tips of the sub-branches. The average $h_{\text {local }}$ for the heat sink "vert" is $6.27 \mathrm{~W} /\left(\mathrm{m}^{2} \cdot \mathrm{K}\right)$. We also summarize the information of the total heat transfer rate via convection and average energy flux via convection in Table 3, which is about $0.89 \mathrm{~W}$ and $35.5 \mathrm{~W} / \mathrm{m}^{2}$, respectively. The radiation dissipates about $0.08 \mathrm{~W}$ and the conduction through the insulation housing is about $0.11 \mathrm{~W}$.

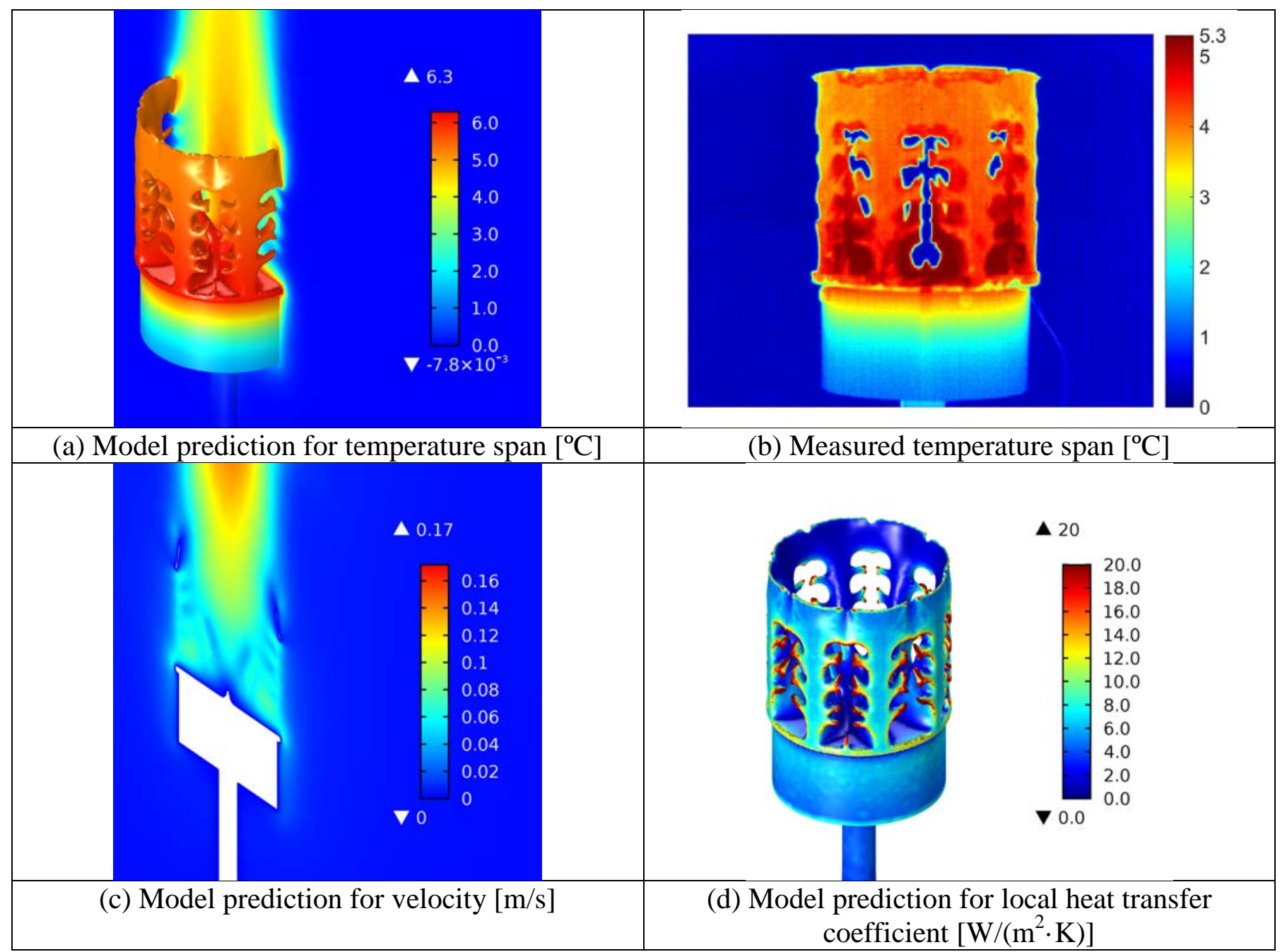

Figure 12. (a) - (b) modelled and measured temperature span, (c) modelled flow velocity and (d) modelled local heat transfer coefficient of the heat sink "vert" with $\alpha=0^{\circ}$ 
Table 3. Parameters of the heat sink surface in the validation simulation

650

\begin{tabular}{|c|c|c|c|c|c|}
\hline & \multicolumn{2}{|c|}{$\alpha=0^{\circ}$} & \multicolumn{3}{|c|}{$\alpha=90^{\circ}$} \\
\hline & "vert" & $\begin{array}{c}\text { "pf- } \\
5.7 \mathrm{~mm} "\end{array}$ & "horiz1" & "horiz2” & $\begin{array}{c}\text { "pf- } \\
5.7 \mathrm{~mm} "\end{array}$ \\
\hline $\begin{array}{c}\text { Total heat transfer rate by } \\
\text { convection [W] }\end{array}$ & 0.89 & 0.88 & 0.90 & 0.90 & 0.88 \\
\hline Average energy flux [W/m²] & 35.5 & 25.2 & 38.7 & 39.1 & 25.4 \\
\hline Average $h_{\text {local }}\left[\mathrm{W} /\left(\mathrm{m}^{2} \cdot \mathrm{K}\right)\right]$ & 6.27 & 4.47 & 6.86 & 6.65 & 4.24 \\
\hline Designed surface area $\left[\mathrm{cm}^{2}\right]$ & 283 & 456 & 266 & 261 & 456 \\
\hline
\end{tabular}

For the heat sink “pf-5.7 mm”, both modelled and measured temperatures in Figure 13(a) and (b) show that the inner pins have a higher temperature than those located further toward the outer edge. The cut pins on the edge have a much lower temperature than those in the middle. From Figure 13(a) and (c), it is observed that the air carrying the heat flows upward like "vert" does. In contrast, the flow velocity at the bottom of "pf-5.7 mm" is very small, meaning the air here flows slowly and it does not transfer heat well with the pins through convection. Figure 13 (a) shows that the surface of the outer pins has a higher $h_{\text {local }}$ than the inner ones, and the average $h_{\text {local }}$ is about $4.47 \mathrm{~W} /\left(\mathrm{m}^{2} \cdot \mathrm{K}\right)$, which is lower than 6.27 $\mathrm{W} /\left(\mathrm{m}^{2} \cdot \mathrm{K}\right)$ for "vert". In addition, the total heat transfer rate of "pf-5.7 mm" is the same as "vert", meaning that the radiation and the conduction loss are almost the same but "pf-5.7 mm" needs a larger temperature span to transfer the same amount of heat. With a larger surface area, the average energy flux of "pf-5.7 mm" is also lower that "vert". The comparison shows the flow guided by the optimized geometry contributes largely to the heat dissipation and the TO design gives a good optimized structure for maximizing performance.

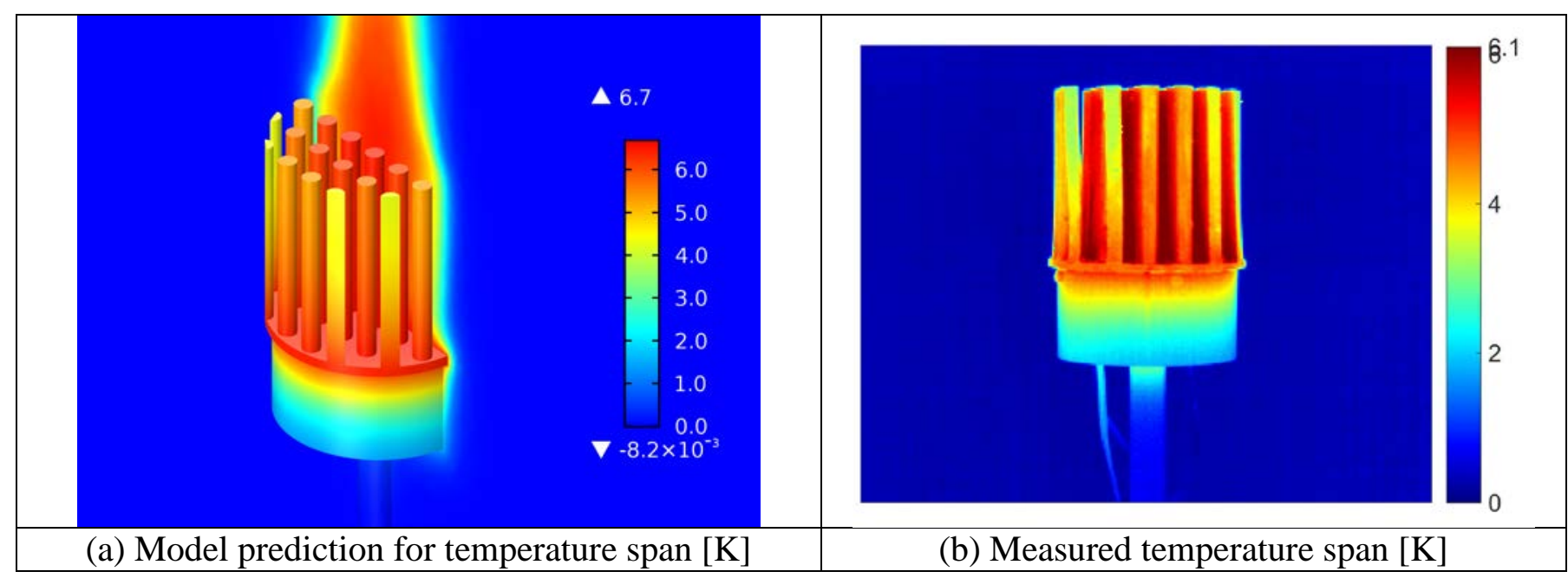




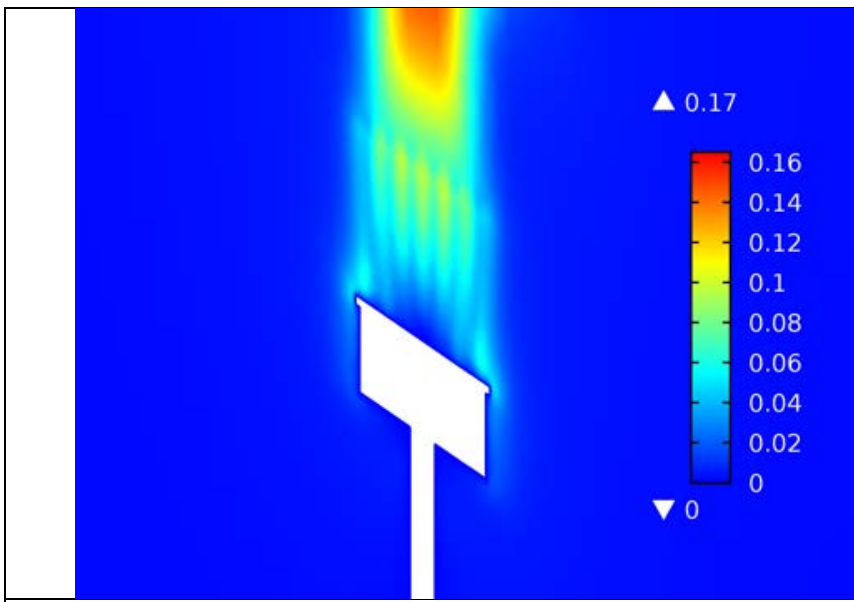

(c) Model prediction for velocity $[\mathrm{m} / \mathrm{s}]$
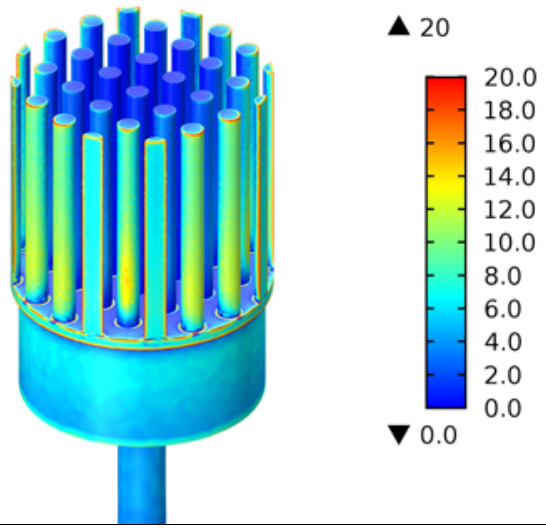

(d) Model prediction for local heat transfer coefficient $\left[\mathrm{W} /\left(\mathrm{m}^{2} \cdot \mathrm{K}\right)\right]$
665

666

667

668

669

670

671

672

673

674

675

676

677

678

679

680

681

682

683

Figure 13. (a) - (b) modelled and measured temperature span, (c) modelled flow velocity and (d) modelled local heat transfer coefficient of the heat sink "pf-5.7mm" with $\alpha=0^{\circ}$

\subsubsection{Analysis of TO and pin fin heat sinks with $\alpha=90^{\circ}$}

For the orientation angle $\alpha=90^{\circ}$, the three heat sinks "horiz1", "horiz2" and "pf-5.7 mm" are presented in detail. The modelled surface temperature distribution in "horiz1" is presented in Figure 14(a). There is a temperature gradient from the base plate to the crown in the heat flux direction. At the same time, the branches at the lower position have a lower temperature than those on the top, as the cold air first contacts and exchanges heat with the branches at the bottom. This behavior is also observed in the measured thermograph in Figure 14(b), although the measured value is lower than the modelled temperature span. As seen in Figure 14(c), the two main branches have a cross section similar to an airplane flap. These branches gather the airflow into the center and the air accelerates due to the buoyancy effect, showing a very efficient geometry for transferring heat by natural convection. The crown of "horiz1" has more sub-branches which dissipate the heat efficiently (see $h_{\text {local }}$ in Figure 14 (d)). A large local heat transfer coefficient is also seen at the lower position. The average $h_{\text {local }}$ is about $6.86 \mathrm{~W} /\left(\mathrm{m}^{2} \cdot \mathrm{K}\right)$. The energy flux is close to that for the heat sinks tested with $\alpha=0^{\circ}$, indicating that the radiation and the conduction loss for different heat sinks are similar.

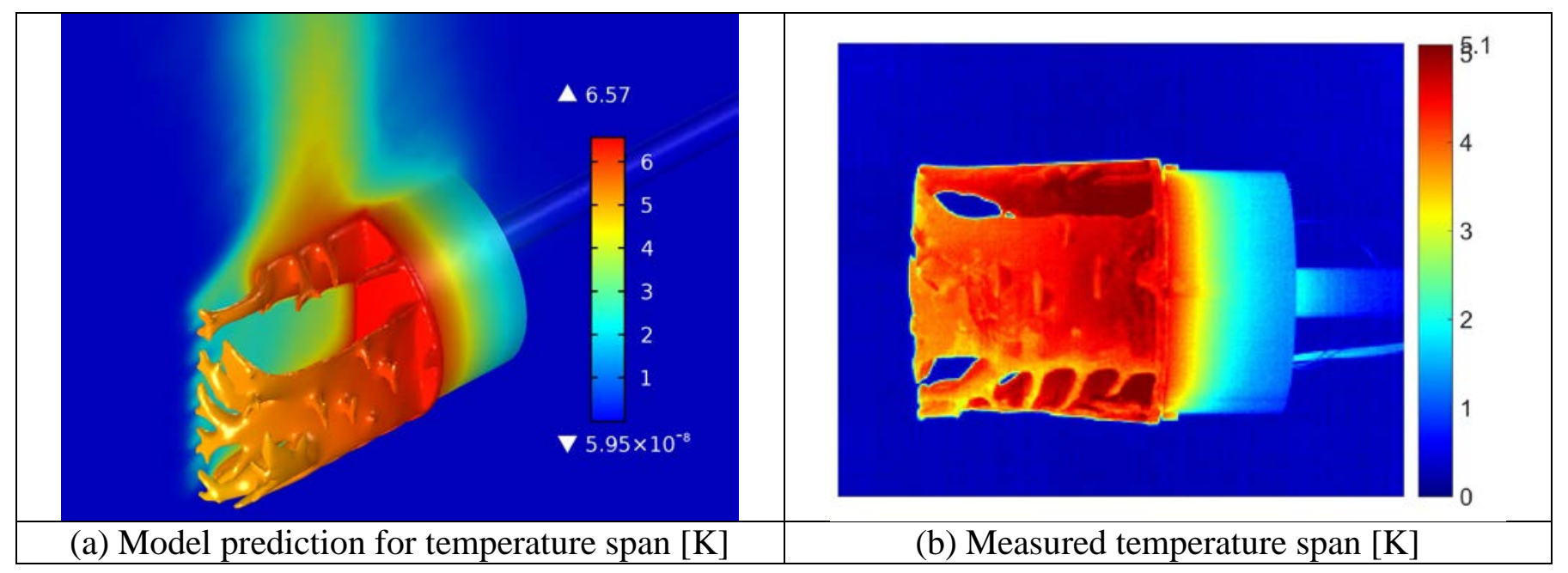




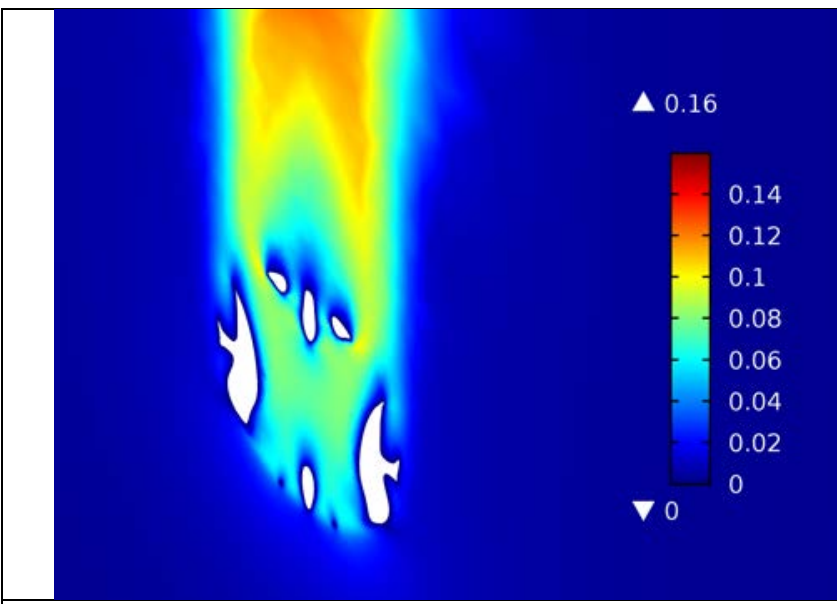

(c) Model prediction for velocity [m/s]

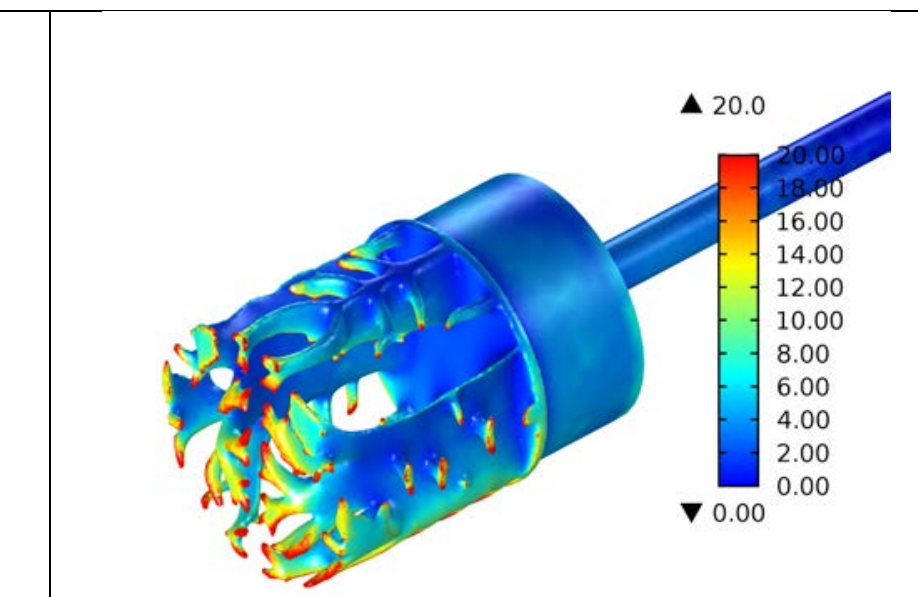

(d) Model prediction for local heat transfer coefficient $\left[\mathrm{W} /\left(\mathrm{m}^{2} \cdot \mathrm{K}\right)\right]$
684

Figure 14. (a) - (b) modelled and measured temperature span, (c) modelled flow velocity and (d) modelled local heat transfer coefficient of the heat sink "horiz1" with $\alpha=90^{\circ}$

Simulation and experimental results of the heat sink "horiz2" are given in Figure 15. As mentioned before, this heat sink is optimized based on a smaller domain, which is $1 / 4$ of the full size. Therefore, the vertical flow is not guided to be fully optimal for heat transfer resulting in a slightly poorer performance than that of "horiz1", as shown in Figure 11(b). Both the temperature distribution predicted by simulation and experimentally measured show that the gradient develops from the base plate to the crown and from lower to higher positions. The flow velocity as shown in Figure 15 (c) explains why this design performs worse than "horiz1". The lee side of the branches in "horiz2" is significantly larger than that of "horiz1", and many branches are not streamlined in the flow direction for this orientation, which blocks natural convection flow paths. This results in a lower air flow through the heat sink compared to "horiz1" which again lowers the overall heat transfer performance of this design. Due to the additional symmetry requirements, not all branches are streamlined in the flow direction, but instead are a form of averaged designs for multiple rotations, as discussed in Section 5.2. Impact of orientation. Although the measured temperature span of "horiz2" is slightly higher than "horiz1", it still shows how the boundary conditions affect the topological design and how the TO designs reflect the physics principles incorporated in the model.

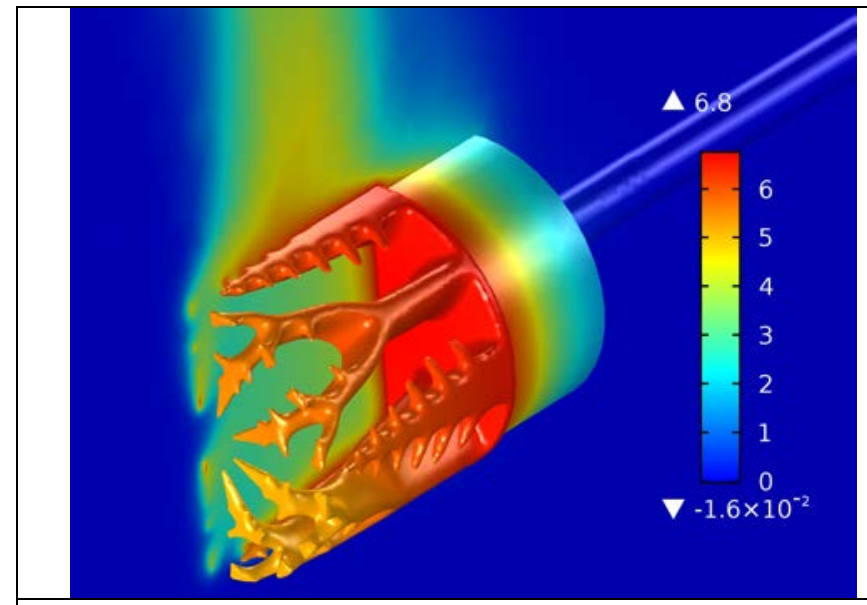

(a) Model prediction for temperature span [K]

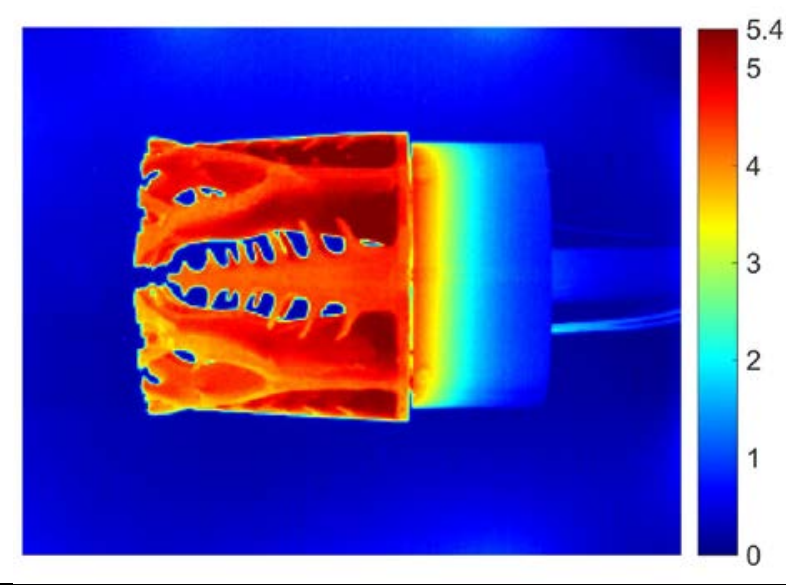

(b) Measured temperature span [K] 


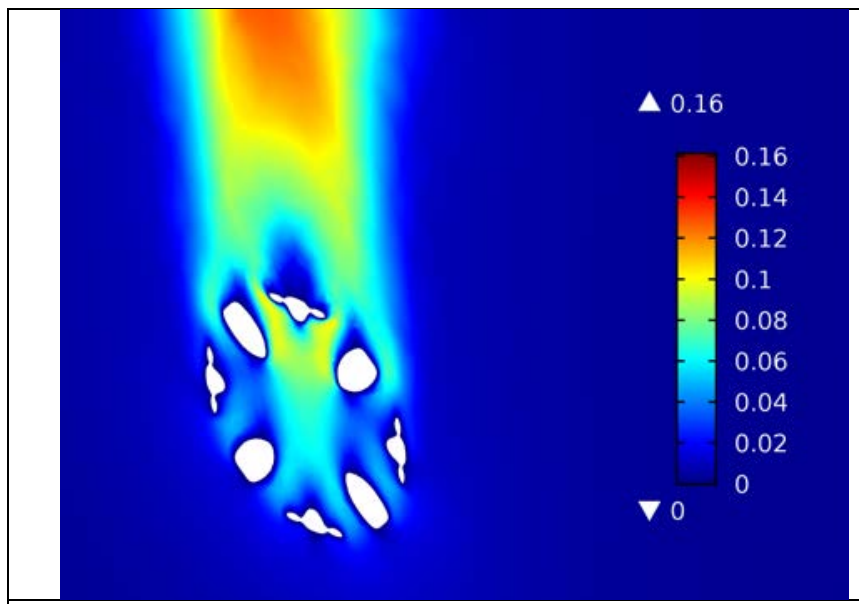

(c) Model prediction for velocity [m/s]

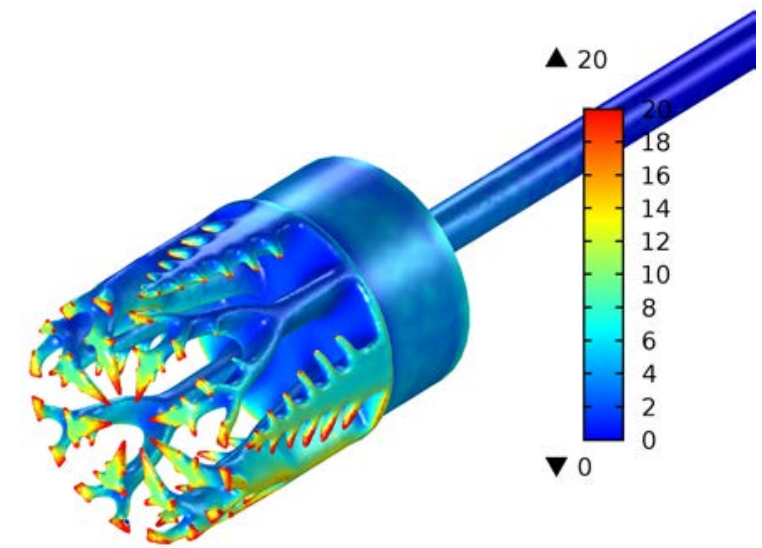

(d) Model prediction for local heat transfer coefficient $\left[\mathrm{W} /\left(\mathrm{m}^{2} \cdot \mathrm{K}\right)\right]$

Figure 15. (a) - (b) modelled and measured temperature span, (c) modelled flow velocity and (d) modelled local heat transfer coefficient of the heat sink "horiz2" with $\alpha=90^{\circ}$

To compare the TO designs to one of the reference heat sinks, simulation and experimental results of the heat sink "pf-5.7 mm" are provided in Figure 16. It can be seen that the behavior of the temperature distribution is similar to that in "horiz1" or "horiz2". Since the experimentally measured temperature span is shown in the side view, the maximum value is much lower than that in the simulation. The flow distribution depicted in Figure 16(c) indicates why this design presents lower performance than the TO designs. Most of the air flows through the gap without good contact with the pins and the velocity inside the heat sink is about $0.06 \mathrm{~m} / \mathrm{s}$, which is much lower than "horiz1" or "horiz2". The local heat transfer coefficient in Figure 16(d) also shows that the pins located at the bottom and outer contact with air well and exhibit a higher local heat transfer coefficient, but many inside do not transfer heat with the air in an efficient way, which is partly due to the high local air temperature. The average local heat transfer coefficient is about $4.24 \mathrm{~W} /\left(\mathrm{m}^{2} \cdot \mathrm{K}\right)$, which is much lower than those for "horiz1" and "horiz2".

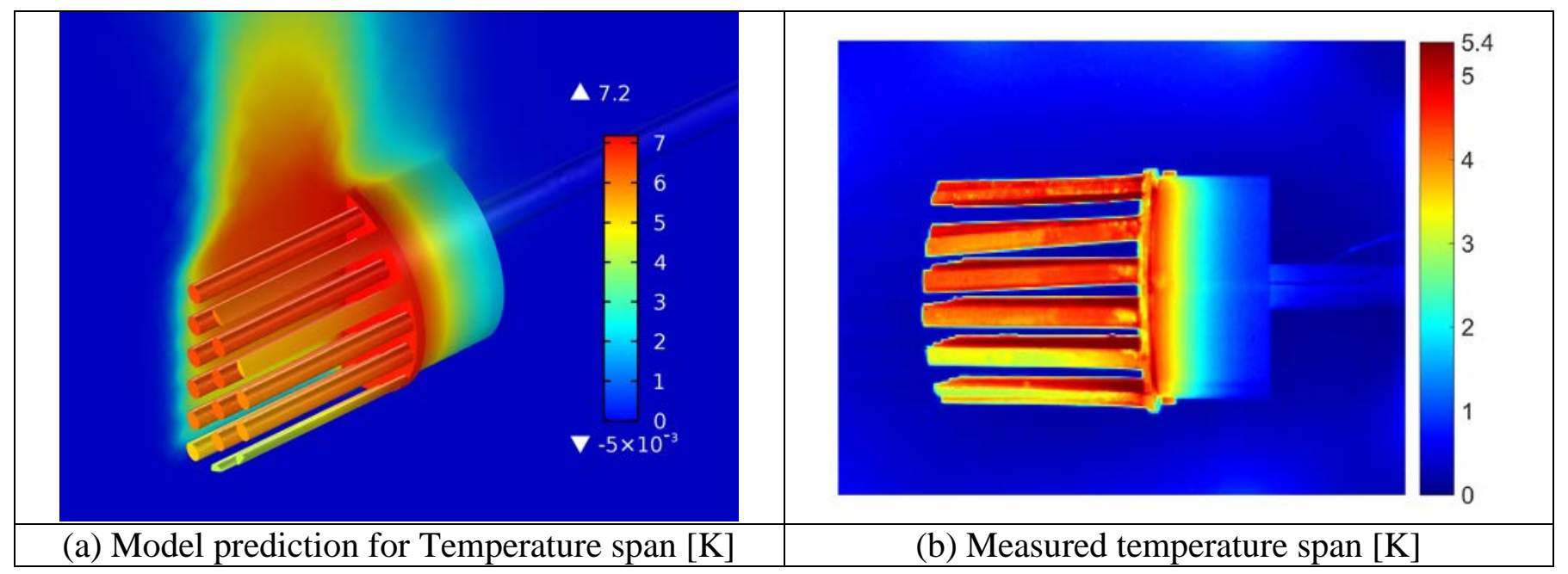




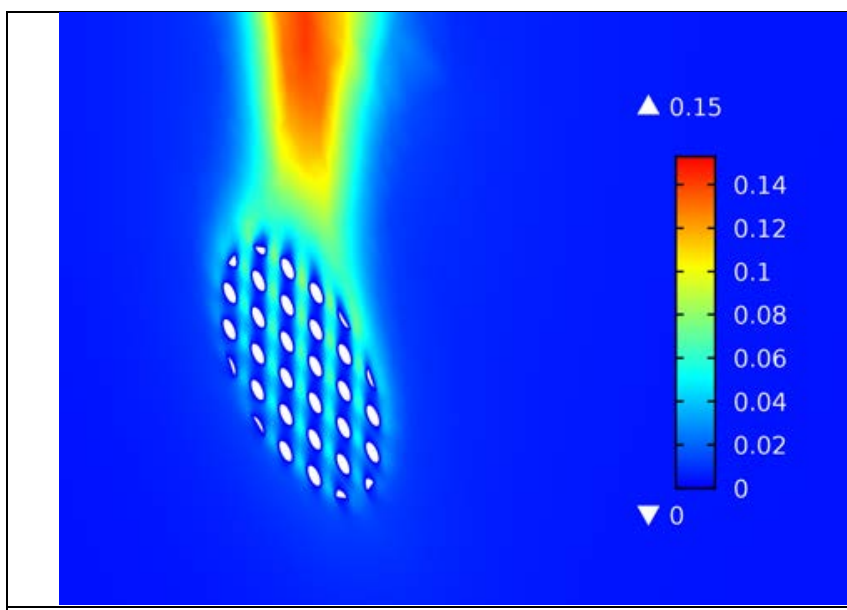

(c) Model prediction for velocity [m/s]

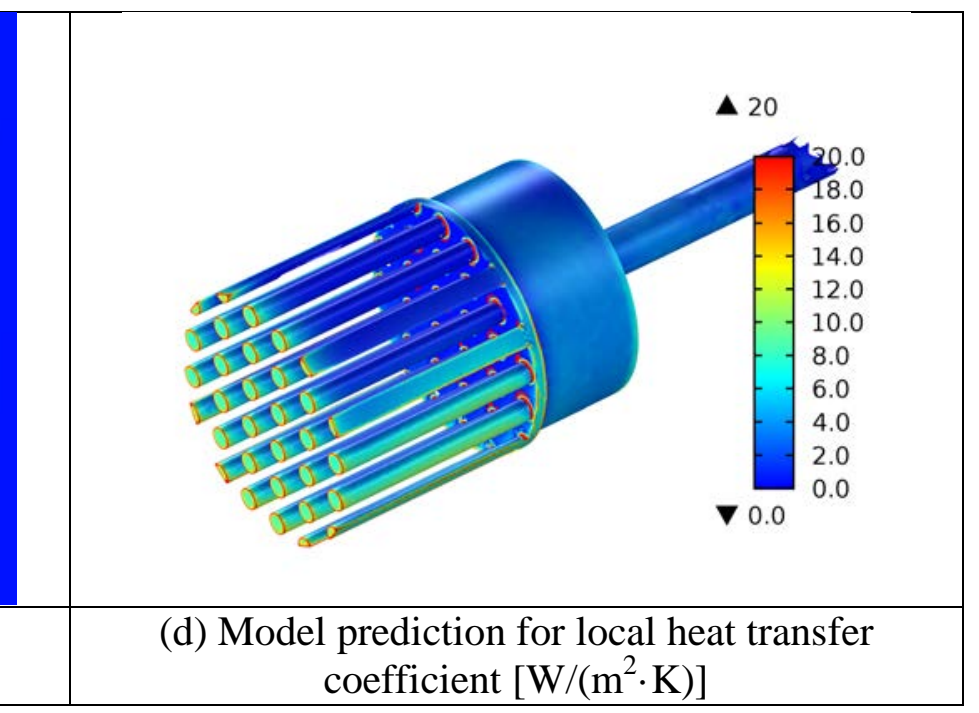

Figure 16. (a) - (b) modelled and measured temperature span, (c) modelled flow velocity and (d) modelled local heat transfer coefficient of the heat sink "pf-5.7mm" with $\alpha=90^{\circ}$

\subsection{Impact of orientation}

The orientation effect with respect to gravity of the TO designs is interesting as they are designed based on different orientation angles, which results in significantly different optimized geometries as shown above. They are supposed to perform best under exactly the working conditions optimized for, but some degradation of performance is expected when the orientation is changed. Therefore, a study on the orientation effect of three heat sinks, "vert", "horiz1" and "pf-5.7mm" is performed and presented in Figure 17. The simulation results are indicated as lines and the experimental results are represented by dots. Repeated experiments generate multiple dots. The simulation shows that the best and the worst designs are "vert" and "horiz1" for the orientation angle $\alpha=0^{\circ}$, and the "pf-5.7mm" is in the middle. When the orientation angle changes to $45^{\circ}$ and $90^{\circ}$, the situation reverses: "horiz1" turns to be the best and "vert" the worst. This shows that the orientation angle has a large impact on the TO designs and the results reflect the preset constraints as predicted. A more robust TO design could be generated using a multiobjective optimization to give a heat sink that performs well at both $\alpha=0^{\circ}$ and $\alpha=90^{\circ}$, but this is not considered here. 


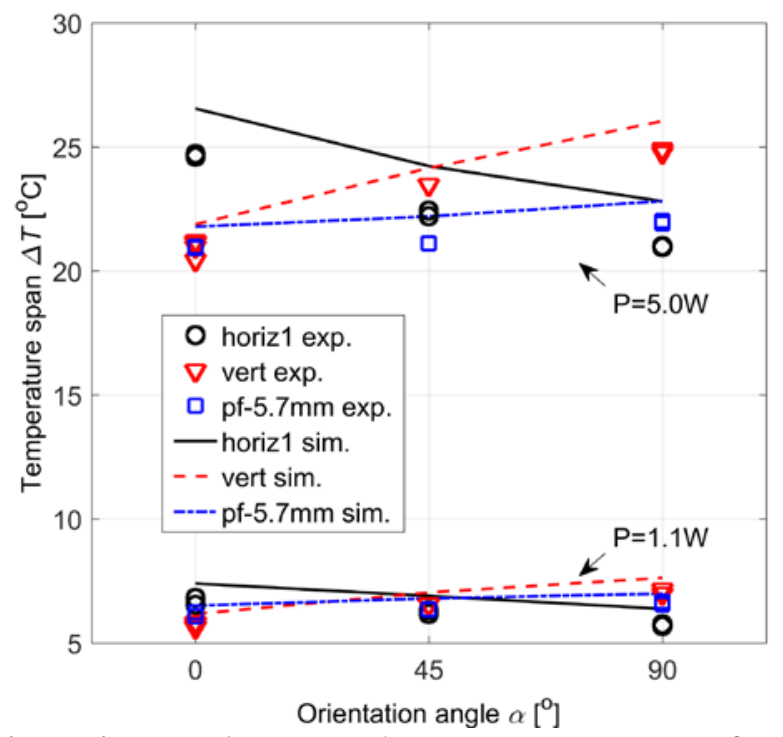

Figure 17. Impact of the orientation angle, $\alpha$, on the temperature span for the three heat sinks "horiz1", "vert" and "pf-5.7mm"

A more detailed study is implemented to further investigate the impact of the rotation angle, or azimuthal angle. Design "horiz1" is chosen, as it was shown to have the best performance at $\alpha=90^{\circ}$, see Figure 11, and because it has two big flap-like branches as seen in Figure 14(c), which could largely affect the flow through the heat sink as it is rotated about its axis. Both simulation and experiment tests start from $\beta=0^{\circ}$, which is the designed rotation angle for "horiz1", and the results are shown in Figure 18. With increasing angle, the streamlined branches turn from the vertical to a more horizontal position, which begin to block the flow. Therefore, the temperature at the aluminum heating plate increases immediately due to decreased air flow through the heat sink. The flap-like branches turn to be horizontal when $\beta=90^{\circ}$, which drives the temperature to reach the maximum temperature. Further increase of $\beta$ turns the branches back to the vertical direction, which lowers the temperature and realizes better heat dissipation performance again. When the angle is $180^{\circ}$, the performance is very close to that for $0^{\circ}$, although the branches are optimized for the reversed direction. The TO heat sinks have been modelled in detail in Ref [38] with regard to orientation and rotation angles, and it was shown that "horiz1" is more sensitive than "horiz2" due to the higher symmetry requirements on optimization of "horiz1". 


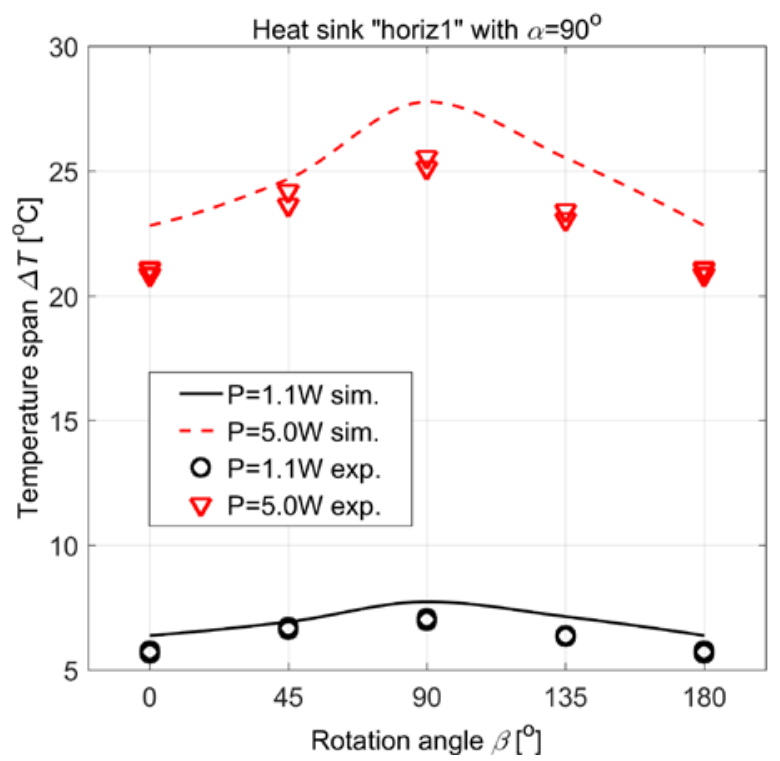

Figure 18. Impact of rotation angle, $\beta$, on temperature span for "horiz1" when the orientation angle, $\alpha$, is $90^{\circ}$

\subsection{Impact of radiation}

As discussed in Section 4.1 and 5.1, the heat sink is painted with graphite spray and the resulting dark black surface gives a radiative emissivity close to 1 , which is preferable for heat dissipation. Although the effect of radiation is not included in the TO model, we present the effect brought by the graphite painting for the TO design "vert" as an exemplary case in Figure 19. With the graphite paint the temperature at the aluminum plate is lower than for an unpainted surface, as expected. The difference increases as the heating power and temperature spans increase, showing a nonlinear behavior. This confirms that such a surface treatment, which has been applied widely in electronics cooling applications, can further improve the heating dissipation performance. 


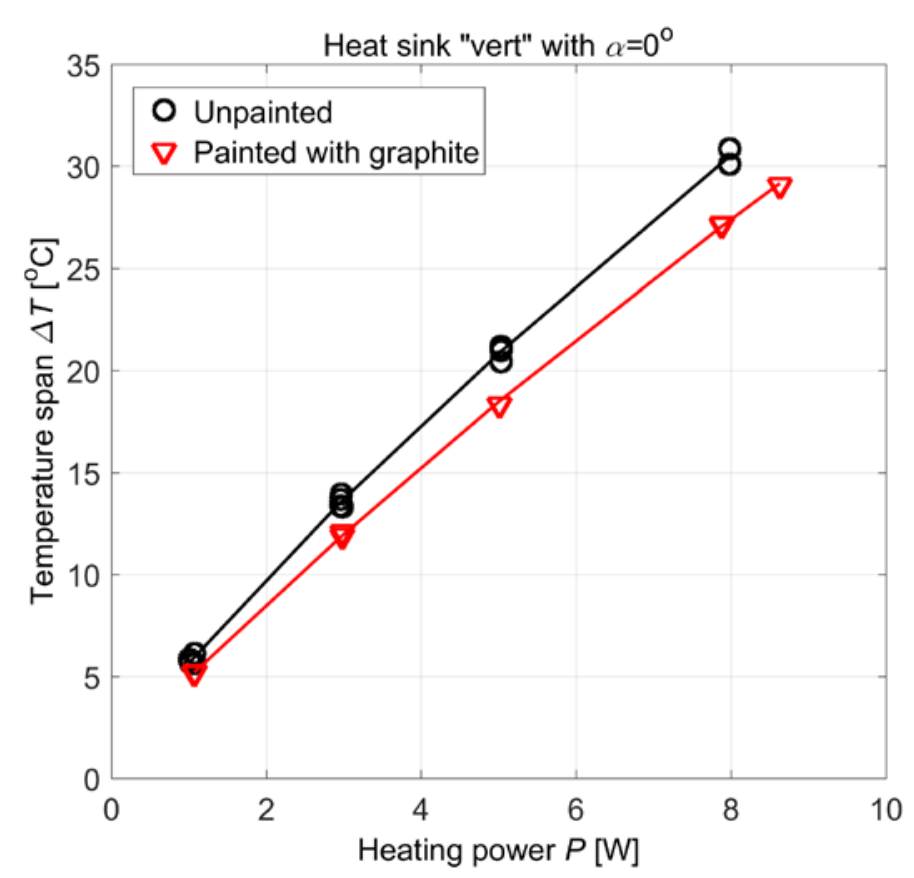

Figure 19. Impact of radiation for the heat sink "vert"

\section{Conclusions}

Stereolithography-assisted investment casting is developed and applied to fabricate metal heat transfer devices designed by topology optimization. A comparison with metal additive manufacturing shows that the SLA-assisted IC technique is a promising alternative, which has a low cost and is flexible with regards to part size and metals that can be used. X-ray micro-tomography was used to check the quality of the cast parts and confirmed that the finished parts were more than $99.9 \%$ dense. The achievable pattern size depends on the capacity of the 3D printing device, however combing multiple wax pieces can increase the maximum cast size for the IC approach.

Building on optimization studies from Refs. [17-20], we present a validation of TO heat sinks by both simulation and experiment. The heat sink "vert" is designed for vertical operation and two more designs, "horiz1" and "horiz2", are generated based on the horizontal directions. Five reference pin-fin heat sinks are also conceived for comparison. Six heat sinks are fabricated based on the SLA-assisted IC technique in Britannia metal and the resulting heat sinks capture structural details with high accuracy. These results successfully demonstrate the capabilities of this combined fabrication process.

A natural convection test apparatus is constructed and simulation models are built in COMSOL Multiphysics for validating the TO heat sink performance. The simulation results fit the experiments well with a variation of less than $8 \%$. Both validation tests show that the TO heat sinks can always realize the best heat dissipation performance at the working conditions optimized for; and even for a higher heating power in some cases. A detailed analysis of the temperature distribution, velocity and local heat transfer coefficients further verifies the high performance of the topological designs, showing the branches generated by TO guide the flow in optimized ways for maximizing the overall heat dissipation performance. Although the heat transfer area is lower compared to the reference heat sinks, optimized geometries still realize lower thermal resistance. Moreover, an orientation study is conducted 
801

802

803

804

805

806

807

808

809

810

811

812

813

814

815

816

817

818

819

820

821

822

823

824

825

826

827

828

829

830

831

832

833

834

835

836

837

838

839

840

841

842

843

844

845

846

847

which shows that the performance of TO heat sinks strongly depends on the design conditions, as predicted. Lastly, the impact of radiation is presented for an exemplarily case.

As the Britannia metal is less conductive than most metals used in heat transfer devices, future studies will emphasize the fabrication and testing of TO designs based on aluminum or copper alloys. A bronze heat sink has been fabricated successfully, which is not presented here, demonstrating that the process can be applied to different metals and alloys. With developments in the TO solvers, more designs can be tested based on this fast prototyping technique with low cost. Please note that this technique is not limited to heat transfer devices but can be applied to other areas such as structural optimization as well.

\section{Acknowledgements}

This work was financed by the TopTEn project sponsored through the Sapere Aude Program of the Danish Council for Independent Research (DFF - 4005-00320). The authors would like to thank Dr. W. R. Kiebach, Dr. K. B. Andersen and Mr. J. Geyti from the Department of Energy Conversion and Storage, Technical University of Denmark, for technical support. The 3D Imaging Centre at The Technical University of Denmark is gratefully acknowledged.

\section{References}

1. M. P. Bendsøe, O. Sigmund, Topology Optimization: Theory, Methods and Applications, Springer, 2003. DOI: http://doi.org/10.1007/978-3-662-05086-6

2. J. D. Deaton, R. V. Grandhi, A survey of structural and multidisciplinary continuum topology optimization: post 2000, Structural and Multidisciplinary Optimization, 2014, 49(1): 1-38. DOI: https://doi.org/10.1007/s00158-013-0956-z

3. O. Sigmund, and K. Maute, Topology optimization approaches A comparative review, Structural and Multidisciplinary Optimization, 2013, 48(6): 1031-1055. DOI: http://doi.org/10.1007/s00158$\underline{\text { 013-0978-6 }}$

4. X. Y. Chen, Topology optimization of microfluidics - A review, Microchemical Journal, 2016, 127: 52-61. DOI: https://doi.org/10.1016/j.microc.2016.02.005

5. T. E. Bruns, Topology optimization of convection-dominated steady-state heat transfer problems, International Journal of Heat and Mass Transfer, 2007, 50: 2859-2873. DOI: https://doi.org/10.1016/j.ijheatmasstransfer.2007.01.039

6. G. H. Yoon, Topological design of heat dissipating structure with forced convective heat transfer, Journal of Mechanical Science and Technology, 2010, 24 (6): 1225-1233. DOI: https://doi.org/10.1007/s12206-010-0328-1

7. G. Marck, M. Nemer and J. L. Harion, Topology optimization of heat and mass transfer problems: laminar flow, Numerical Heat Transfer Part B: Fundamentals, 2013, 63 (6): 508-539. DOI: http://dx.doi.org/10.1080/10407790.2013.772001

8. P. Coffin, and K. Maute, Level set topology optimization of cooling and heating devices using a simplified convection model, Structural and Multidisciplinary Optimization, 2016, 53(5): 985-1003. DOI: https://doi.org/10.1007/s00158-015-1343-8

9. E. M. Dede, Experimental investigation of the thermal performance of a manifold hierarchical microchannel cold plate, Proceedings of the ASME Pacific Rim Technical Conference \& Exposition on Packaging and Integration of Electronic and Photonic Systems, Portland, USA, 2011.

10. E. M. Dede and Y. Liu, Experimental and numerical investigation of a multi-pass branching microchannel heat sink. Applied Thermal Engineering, 2013, 55:51-60. 
848

849

850

851

852

853

854

855

856

857

858

859

860

861

862

863

864

865

866

867

868

869

870

871

872

873

874

875

876

877

878

879

880

881

882

883

884

885

886

887

888

889

890

891

892

893
11. E. M. Dede, Single-Phase Microchannel Cold Plate for Hybrid Vehicle Electronics, Semiconductor Thermal Measurement and Management Symposium (SEMI-THERM), San Jose, USA, 2014.

12. A. A. Koga, E. C. C. Lopes, H. F. V. Nova, et al., Development of heat sink device by using topology optimization. International Journal of Heat and Mass Transfer, 2013, 64: 759-772. DOI: http://doi.org/10.1016/j.ijheatmasstransfer.2013.05.007

13. E. M. Dede, Multiphysics topology optimization of heat transfer and fluid flow systems, Proceedings of the COMSOL Conference, Boston, USA, 2009.

14. COMSOL Multiphysics ${ }^{\circledR}$ Modeling Software, https://www.comsol.com/, September 19, 2017.

15. E. M. Dede, S. N. Joshi and F. Zhou, Topology optimization, additive layer manufacturing, and experimental testing of an air-cooled heat sink, J. Mech. Des. 2015, 137 (111702): 1-9. DOI: https://doi.org/10.1115/1.4030989

16. J. H. K. Haertel and G. F. Nellis, A fully developed flow thermofluid model for topology optimization of 3D-printed air-cooled heat exchangers, Applied Thermal Engineering, 119: 10-24. DOI: https://doi.org/10.1016/j.applthermaleng.2017.03.030

17. J. Alexandersen, N. Aage, C. S. Andreasen, et al., Topology optimisation for natural convection problems, International Journal for Numerical Methods in Fluids, 2014, 76 (10): 699-721. DOI: http://dx.doi.org/10.1002/fld.3954

18. J. Alexandersen, O. Sigmund and N. Aage, Large scale three-dimensional topology optimisation of heat sinks cooled by natural convection. International Journal of Heat and Mass Transfer, 2016, 100 : 876-891. DOI: http://doi.org/10.1016/j.ijheatmasstransfer.2016.05.013

19. J. Alexandersen, O. Sigmund and N. Aage, Topology optimisation of passive coolers for lightemitting diode lamps, The $11^{\text {th }}$ World Congress on Structural and Multidisciplinary Optimization, Sydney, Australia, 2015.

20. B. S. Lazarov, O. Sigmund, K. E. Meyer, and J. Alexandersen, Experimental validation of additively manufactured optimized shapes for passive cooling, Applied Energy, 2018, 226: 330-339. DOI: 10.1016/j.apenergy.2018.05.106.

21. S. B. Dilgen, C. B. Dilgen, D. R. Fuhrman, O. Sigmund, B. S. Lazarov, Density Based Topology Optimization of Turbulent Flow Heat Transfer System. Structural and Multidisciplinary Optimization, available online. DOI: https://doi.org/10.1007/s00158-018-1967-6

22. S. Singh and R. Singh, Precision investment casting: A state of art review and future trends. Proceedings of the Institution of Mechanical Engineers Part B: Journal of Engineering Manufacture, 2016, 230 (12): 2143-2164. DOI: http://dx.doi.org/10.1177/0954405415597844

23. Jewelry Resins for Superb Detail, https://formlabs.com/materials/jewelry/, September 19, 2017.

24. 3D Hubs, https://www.3dhubs.com/, September 19, 2017.

25. E. O. Olakanmi, R. F. Cochrane and K. W. Dalgarno, A review on selective laser sintering/melting (SLS/SLM) of aluminium alloy powders: Processing, microstructure, and properties. Progress in Materials Science, 2015, 74: 401-477. http://doi.org/10.1016/j.pmatsci.2015.03.002

26. N. T. Aboulkhair, N. M. Everitt, I. Ashcroft, et al., Reducing porosity in AlSi10Mg parts processed by selective laser melting. Additive Manufacturing, 2014, 1-4: 77-86. DOI: http://doi.org/10.1016/j.addma.2014.08.001.

27. Yang, T. Lu and T. Kim, Effective thermal conductivity modelling for closed-cell porous media with analytical shape factors. Transp. Porous Med., 2013, 100: 211-224. DOI: http://dx.doi.org/10.1007/s11242-013-0212-4

28. J. P. M. Florez, M. B. H. Mantelli and G. G. V. Nuernberg, Effective thermal conductivity of sintered porous media: model and experimental validation. International Journal of Heat and Mass Transfer, 2013, 66: 868-878. DOI: http://dx.doi.org/10.1016/j.ijheatmasstransfer.2013.07.088 
894

895

896

897

898

899

900

901

902

903

904

905

906

907

908

909

910

911

912

913

914

915

916

917

918

919

920

921

922

923

924

29. B. S. Lazarov and O. Sigmund, Filters in topology optimization based on Helmholtz-type differential equations, International Journal for Numerical Methods in Engineering, 2011, 86(6): 765-781. DOI: 10.1002/nme.3072

30. B. S. Lazarov, F. Wang, and O. Sigmund, Length scale and manufacturability in density-based topology optimization, Archive of Applied Mechanics, 2016, 86(1): 189-218. DOI: https://doi.org/10.1007/s00419-015-1106-4

31. R. Bahadur and A. Bar-Cohen, Thermal Design and Optimization of Natural Convection Polymer Pin Fin Heat Sinks, IEEE Transactions on Components and Packaging Technologies, 2005, 28(2): 238-246.

32. Y. Yang and H. Peng, Numerical study of pin-fin heat sink with un-uniform fin height design, International Journal of Heat and Mass Transfer, 2008, 51: 4788-4796.

33. N. Aage, E. Andreassen, and B. Lazarov, Topology optimization using petsc: An easy-to-use, fully parallel, open source topology optimization framework, Structural and Multidisciplinary Optimization, 2015, 51(3): 565-572. DOI: https://doi.org/10.1007/s00158-014-1157-0

34. N. Aage and B. Lazarov, Parallel framework for topology optimization using the method of moving symptotes, Structural and Multidisciplinary Optimization, 2013, 47(4): 493-505. DOI: https://doi.org/10.1007/s00158-012-0869-2

35. K. Svanberg, The method of moving asymptotes - a new method for structural optimization, International Journal for Numerical Methods in Engineering, 1987, 24: 359-373. DOI: 10.1002/nme.1620240207

36. A. I. Zografos and J. E. Sunderland, Natural convection from pin fin arrays, Experimental Thermal and Fluid Science, 1990, 3 (4): 440-449. DOI: https://doi.org/10.1016/0894-1777(90)90042-6

37. Using the Boussinesq Approximation for Natural Convection, https://www.comsol.com/blogs/usingthe-boussinesq-approximation-for-natural-convection/, September 19, 2017.

38. J. Alexandersen, O. Sigmund, K. E. Meyer, B. S. Lazarov, Design of passive coolers for lightemitting diode lamps using topology optimisation, International Journal of Heat and Mass Transfer, 2018, 122: 138-149. DOI: https://doi.org/10.1016/j.ijheatmasstransfer.2018.01.103.

39. J. P. Gwinn and R. L. Webb, Performance and testing of thermal interface materials, Microelectronics Journal, 2003, 34: 215-222.

40. H. Minseok and S. Graham, Development of a thermal resistance model for chip-on-board packaging of high power LED arrays, Microelectronics Reliability, 2012, 52: 836-844. 